\title{
A Riemannian interpolation inequality à la Borell, Brascamp and Lieb
}

\section{Dario Cordero-Erausquin ${ }^{1}$, Robert J. McCann ${ }^{2, \star}$, Michael Schmuckenschläger $^{3}$}

1 Equipe d'Analyse et de Mathématiques Appliquées, Université de Marne-la-Vallée, 77454 Marne-la-Vallée Cedex 2, France (e-mail: cordero@math.univ-mlv. fr)

2 Department of Mathematics, University of Toronto, Toronto Ontario Canada M5S 3G3 (e-mail: mccann@math. toronto.edu)

3 Institut für Analysis und Numerik, Universität Linz, A-4040 Linz, Österreich (e-mail: michael.schmuckenschlaeger@telering.at)

Oblatum 1-XII-2000 \& 3-V-2001

Published online: 13 August 2001 - (C) Springer-Verlag 2001

\begin{abstract}
A concavity estimate is derived for interpolations between $L^{1}(M)$ mass densities on a Riemannian manifold. The inequality sheds new light on the theorems of Prékopa, Leindler, Borell, Brascamp and Lieb that it generalizes from Euclidean space. Due to the curvature of the manifold, the new Riemannian versions of these theorems incorporate a volume distortion factor which can, however, be controlled via lower bounds on Ricci curvature. The method uses optimal mappings from mass transportation theory. Along the way, several new properties are established for optimal mass transport and interpolating maps on a Riemannian manifold.
\end{abstract}

\section{Contents}

1 Introduction and main results . . . . . . . . . . . . . . . . . 220

2 Riemannian geometry: exponential map, distance functions, and volume distortion coefficients . . . . . . . . . . . . . . . . . . . 227

3 Background on mass transport and properties of $c$-concave functions . . . . . . 234

4 The optimal transport Jacobian . . . . . . . . . . . . . . . . . . . . . . . . 241

5 Optimal interpolating maps and densities . . . . . . . . . . . . . . . . . 247

6 Proof of Main Theorem and its corollaries . . . . . . . . . . . . . . . . 251

* RJM gratefully acknowledges the hospitality of the Georgia Institute of Technology and the Max-Planck Institut für Mathematik in den Naturwissenschaften during various stages of this work. His research was supported by Natural Sciences and Engineering Research Council of Canada grant 217006-99 RGPIN and National Science Foundation grant DMS 0074037.

Reproduction of this article, in its entirety, is permitted for non-commercial purposes. 


\section{Introduction and main results}

Functional versions of geometric inequalities have often proved fruitful in geometric analysis. A celebrated example is the functional version of the Brunn-Minkowski inequality, discovered by Prékopa [29,30] and Leindler [23]. Given three non-negative functions $f, g, h: \mathbf{R}^{n} \rightarrow \mathbf{R}_{+}$and $t \in$ $[0,1]$, Prékopa and Leindler proved that when

$$
h((1-t) x+t y) \geq f^{1-t}(x) g^{t}(y)
$$

holds for every $x, y \in \mathbf{R}^{n}$, then $\int_{\mathbf{R}^{n}} h \geq\left(\int_{\mathbf{R}^{n}} f\right)^{1-t}\left(\int_{\mathbf{R}^{n}} g\right)^{t}$.

Applying this inequality to indicator functions of sets $A, B \subset \mathbf{R}^{n}$ one recovers, after suitable scaling, the Brunn-Minkowski inequality:

$$
\operatorname{vol}^{1 / n}[(1-t) A+t B] \geq(1-t) \operatorname{vol}^{1 / n} A+t \operatorname{vol}^{1 / n} B .
$$

First derived in connection with the isoperimetric theorem, applications of (1) pervade convex geometry; see e.g. Schneider [34] or Gardner [18]. But the Prékopa-Leindler inequality has many other implications, including the preservation of logarithmic concavity under convolutions, noticed by Brascamp and Lieb [12] in connection with their proof that diffusion in a convex potential preserves log-concavity [11]. It has similarly been used in the setting of Gaussian-like measures by Maurey [24], Bobkov and Ledoux [8] to derive concentration of measure, Poincaré and logarithmic Sobolev inequalities.

Independently, Borell [10], Brascamp and Lieb [12] obtained generalizations of the Prékopa-Leindler inequality to other means. For $p \in \overline{\mathbf{R}}$, $t \in[0,1]$, and non-negative reals $a, b$, introduce the $p$-mean

$$
\mathcal{M}_{t}^{p}(a, b):=\left((1-t) a^{p}+t b^{p}\right)^{1 / p}
$$

if $a b \neq 0$ and $\mathcal{M}_{t}^{p}(a, b)=0$ if $a b=0$. The cases $p \in\{0, \pm \infty\}$ are defined as limits: $\mathcal{M}_{t}^{-\infty}(a, b):=\min \{a, b\}, \mathcal{M}_{t}^{+\infty}(a, b):=\max \{a, b\}$, while $\mathcal{M}_{t}^{0}(a, b):=a^{1-t} b^{t}$ coincides with the geometric mean. The BorellBrascamp-Lieb inequality asserts that whenever $p \geq-1 / n, t \in[0,1]$, and non-negative functions $f, g, h: \mathbf{R}^{n} \rightarrow \mathbf{R}_{+}$satisfy

$$
h((1-t) x+t y) \geq \mathcal{M}_{t}^{p}(f(x), g(y))
$$

for every $x, y \in \mathbf{R}^{n}$, then

$$
\int_{\mathbf{R}^{n}} h \geq \mathcal{M}_{t}^{p /(1+n p)}\left(\int_{\mathbf{R}^{n}} f, \int_{\mathbf{R}^{n}} g\right) .
$$

In one-dimension, similar inequalities were anticipated by Henstock and Macbeath [21]; (the reviews by Das Gupta [15] or Gardner [18] offer more 
historical commentary). If $p=0$ one recovers the Prékopa-Leindler inequality. Brascamp and Lieb also observed that (2) need only to be satisfied almost everywhere in the sense that it can be replaced by

$$
h(z) \geq \operatorname{esssup}_{z=(1-t) x+t y} \mathcal{M}_{t}^{p}(f(x), g(y)) .
$$

The strongest inequality is obtained when $p=-1 / n$. Indeed, the inequalities for other $p$-means can be derived from this extremal case by using elementary properties of $\mathcal{M}_{t}^{p}$. It is convenient to formulate the case $p=-1 / n$ in the following way, for which we give a physical interpretation after (7). Given $t \in[0,1]$ and three non-negative functions $f, g$ and $h$ on $\mathbf{R}^{n}$ with $\int f=\int g=1$, if

$$
\frac{1}{h((1-t) x+t y)^{1 / n}} \leq(1-t) \frac{1}{f(x)^{1 / n}}+t \frac{1}{g(y)^{1 / n}}
$$

for every $x, y \in \mathbf{R}^{n}$, then $\int_{\mathbf{R}^{n}} h \geq 1$.

The aim of our work is to generalize the above inequalities to curved geometries. The first named author previously obtained [13] such generalizations for spherical and hyperbolic geometries. His proof, based on McCann's optimal mass transport on manifolds [27], assumed various properties of mass transport that will be established in the present paper. As in the Euclidean case [25] [26], the strategy here and there was to use an optimal map to localize geometrical inequalities under an integral, thus reducing them to algebraic relations such as the concavity of $\operatorname{det}^{1 / n}(\cdot)$ on positive matrices, or equivalently, domination of the geometric by the arithmetic mean. We stress that the functional inequalities discussed in this paper are interpolation inequalities: aside from taking place on more general manifolds, they have quite different content from the set enlargement and isoperimetric inequalities by Schmidt [33] on the hyperbolic space and by Gromov [20] on compact positively curved manifolds.

Unless otherwise stated, $M$ or $M^{n}$ denote a complete, connected, $n$ dimensional manifold equipped with a Riemannian metric tensor given by $\mathcal{C}^{2}$-smooth functions $g_{i j}$ in coordinates. We abbreviate all these hypothesis by saying $M$ is continuously curved, since the $\mathcal{C}^{2}$-smoothness implies its Riemann curvature tensor varies continuously from point to point. The geodesic distance between $x$ and $y \in M$ is denoted by $d(x, y)$ and the Riemannian element of volume by $d \operatorname{vol}(x)\left(=\sqrt{\operatorname{det} g_{i j}(x)} d^{n} x\right.$ in coordinates). For $x \in M$, the scalar product and the norm on the tangent space $T_{x} M$ will be denoted by $\langle\cdot, \cdot\rangle$ and $|\cdot|$, respectively; we hope the implicit dependence on $x$ will always be clear from context.

The first task is to define a notion of barycenter to play the role of $(1-t) x+t y$. For fixed $t \in[0,1]$, define the locus of points

$$
Z_{t}(x, y):=\{z \in M \mid d(x, z)=t d(x, y) \text { and } d(z, y)=(1-t) d(x, y)\}
$$


lying partway between $x$ and $y \in M$. When the minimal geodesic $\gamma$ : $[0,1] \longrightarrow M$ linking $x$ to $y$ is unique, then $Z_{t}(x, y)=\{\gamma(t)\}$ consists of the single point dividing this geodesic into segments with ratio $t:(1-t)$. Extend the definition to sets $Y \subset M$ analogously:

$$
Z_{t}(x, Y):=\bigcup_{y \in Y} Z_{t}(x, y)
$$

Letting $B_{r}(y) \subset M$ denote the open ball of radius $r>0$ centered at $y \in M$, for $t \in(0,1]$ we introduce the ratio which plays a crucial role in our study:

$$
v_{t}(x, y):=\lim _{r \rightarrow 0} \frac{\operatorname{vol}\left[Z_{t}\left(x, B_{r}(y)\right)\right]}{\operatorname{vol}\left[B_{t r}(y)\right]}>0 .
$$

This ratio measures the volume distortion due to curvature along the shortest path joining $x$ to $y$; the limit (6) always exists, though it will be infinite when $x$ and $y$ are conjugate points. In any case $v_{1}(x, y)=1$. If light travels on geodesics, then $v_{0}(x, y):=\lim _{t \rightarrow 0} v_{t}(x, y)$ represents the area magnification observed at $x$ of a small light source located near $y$, due to the lensing effects of curvature. Thus $v_{t}(x, y) \geq 1$ if the curvature is non-negative, while the opposite inequality holds in spaces of non-positive curvature. By Thales theorem, $v_{t}(x, y)=1$ in Euclidean space.

Main Theorem (A Riemannian Borell-Brascamp-Lieb inequality) Let $M$ be a continuously curved, $n$-dimensional Riemannian manifold. Fix $t \in$ $[0,1]$. Let $f, g, h \geq 0$ be non-negative functions on $M$, and $A, B$ be Borel subsets of $M$ for which $\int_{A} f=\int_{B} g=1$. Assume that for every $(x, y) \in$ $A \times B$ and every $z \in Z_{t}(x, y)$,

$$
\frac{1}{h(z)^{1 / n}} \leq(1-t)\left[\frac{v_{1-t}(y, x)}{f(x)}\right]^{1 / n}+t\left[\frac{v_{t}(x, y)}{g(y)}\right]^{1 / n} .
$$

Then $\int_{M} h \geq 1$.

Condition (7) should be compared to (4). The non-Euclidean character of $M$ enters through the volume distortion coefficients $v_{t}$. Note that $v_{t}(x, y)^{1 / n}$ is a geometric mean of the linear magnification factors, while $h^{-1 / n}$ scales linearly with distance. Imagining $f, g$ and $h$ to represent densities of three compressible fluids on the manifold, our Main Theorem specifies a local upper bound for the mean separation of the molecules of $h$ to conclude their aggregate mass exceeds the total amount of $f$ and $g$; (to pack molecules of $h$ tightly throughout space requires that lots of them be present).

As in the Euclidean case, algebraic inequalities relating the different $p$-means lead to immediate generalizations of the whole family of BorellBrascamp-Lieb inequalities: 
Corollary 1.1 (Riemannian $p$-Mean inequalities) Let $M$ be a continuously curved, $n$-dimensional Riemannian manifold. Fix $p \geq-1 / n$ and $t \in[0,1]$. Let $f, g, h \geq 0$ be non-negative functions, and $A, B$ be Borel subsets of $M$ carrying the full mass of $f$ and $g$, respectively. Assume that for every $(x, y) \in A \times B$ and every $z \in Z_{t}(x, y)$,

$$
h(z) \geq \mathcal{M}_{t}^{p}\left(\frac{f(x)}{v_{1-t}(y, x)}, \frac{g(y)}{v_{t}(x, y)}\right) .
$$

Then

$$
\int_{M} h \geq \mathcal{M}_{t}^{p /(1+n p)}\left(\int_{M} f, \int_{M} g\right)
$$

For $p=0$ we obtain a Riemannian version of the Prékopa-Leindler inequality. One is free to take $A=B=M$, but since $A$ and $B$ may merely be sets of full measure, it is interesting to note that hypothesis (8) need only be satisfied almost everywhere; the essential supremum condition (3) of Brascamp and Lieb comes built into our formulation automatically. It must also be noted that in Euclidean space, one can go back and deduce the inequality for the mean $\mathcal{M}_{t}^{-1 / n}$ from the inequality for any other $p>-1 / n$. This is done by exploiting the homogeneity of Lebesgue measure. In the Riemannian setting, the lack of homogeneity presumably implies that the inequality for $p=-1 / n$ is genuinely stronger than the inequalities for other $p$-means.

The hypotheses of these theorems become easier to check if one is able to compute the coefficients $v_{t}$ explicitly, or at least provide explicit bounds from below. This can be achieved whenever the Ricci curvature is known to be bounded below: Ric $\geq(n-1) k$, an abbreviation signifying non-negativity of the quadratic form $\operatorname{Ric}(\cdot, \cdot)-k(n-1)\langle\cdot, \cdot\rangle$. Denote by $\mathbf{S}^{n}$ and $\mathbf{H}^{n}$ the $n$-dimensional sphere and hyperbolic space, respectively, of constant sectional curvature \pm 1 , hence Ricci curvature $\pm(n-1)$. Introduce for $k \in \mathbf{R}$,

$$
S_{k}(d):=\frac{\sin (\sqrt{k} d)}{\sqrt{k} d}=\left\{\begin{array}{cll}
(\sin d) / d & \text { for } k=1 & \left(\mathbf{S}^{n}\right) \\
1 & \text { for } k=0 & \left(\mathbf{R}^{n}\right) \\
(\sinh d) / d & \text { for } k=-1 & \left(\mathbf{H}^{n}\right)
\end{array} .\right.
$$

If $M_{k}^{n}$ has constant sectional curvature $k \in \mathbf{R}$, we easily verify

$$
v_{t}(x, y)=\left(\frac{S_{k}(t d(x, y))}{S_{k}(d(x, y))}\right)^{n-1} .
$$

If $M^{n}$ satisfies Ric $\geq(n-1) k$ then, as explained below, Bishop's comparison theorem [7] yields a bound for the volume distortion along any geodesic of length $d(x, y)$ :

$$
v_{t}(x, y) \geq\left(\frac{S_{k}(t d(x, y))}{S_{k}(d(x, y))}\right)^{n-1} .
$$


It asserts that this distortion is minimized in the model space $\mathbf{S}^{n}, \mathbf{R}^{n}$ or $\mathbf{H}^{n}$ of constant sectional curvature k. One can therefore rewrite the BorellBrascamp-Lieb inequalities more quantitatively by assuming a lower bound for the Ricci curvature. For instance in the Prékopa-Leindler case:

Corollary 1.2 (A Riemannian Prékopa-Leindler inequality) Let $M$ be a continuously curved, $n$-dimensional Riemannian manifold on which Ric $\geq$ $(n-1) k$ holds for some $k \in \mathbf{R}$. Fix $t \in[0,1]$. Let $f, g, h: M \longrightarrow \mathbf{R}_{+}$be non-negative functions on $M$, and $A, B$ be Borel subsets of $M$ carrying the full mass of $f$ and $g$, respectively. If every $(x, y) \in A \times B, z \in Z_{t}(x, y)$ and $d:=d(x, y)$ satisfy

$$
h(z) \geq\left(\frac{S_{k}(d)}{S_{k}^{1-t}((1-t) d) S_{k}^{t}(t d)}\right)^{n-1} f^{1-t}(x) g^{t}(y),
$$

then

$$
\int_{M} h \geq\left(\int_{M} f\right)^{1-t}\left(\int_{M} g\right)^{t}
$$

For the sphere and hyperbolic space, Cordero-Erausquin's inequalities [13] are recovered. If $k$ instead bounds the sectional curvatures of $M$ from above, Gunther's comparison theorem implies the reverse of (11) but this inequality is not particularly useful in the present context.

The idea behind our proof admits a physical interpretation, which has roots in the work of McCann [25,26] on $\mathbf{R}^{n}$ and Cordero-Erausquin [13] on the sphere and hyperbolic space. Imagining $\rho_{0}:=f$ and $\rho_{1}:=g$ to represent the initial and final distributions of a compressible fluid throughout the manifold, the idea is to construct a dynamical path $\rho_{t}$ joining $\rho_{0}$ to $\rho_{1}$ along which all fluid particles move geodesically. Mass conservation yields $\left\|\rho_{t}\right\|_{1}=1$, so if the path satisfies $\rho_{t} \leq h$ at the appropriate time the theorem follows immediately. To achieve this bound, we would like to keep the fluid particles as spread out as possible at each time along the path $\rho_{t}$. Positive curvature facilitates this task while negative curvature impedes it - accounting for the distortion factors $v_{t}(x, y)$ in (7) which level out the playing field. However, we still must prevent the fluid particles from focusing at any instant in space and time.

This goal can be realized in one of several equivalent ways, corresponding roughly to the Eulerian versus Lagrangian choices of fluid variables. The Eulerian approach would be to select a path $(\rho, P): M \times[0,1] \longrightarrow$ $[0, \infty] \times T M$ minimizing Benamou and Brenier's action integral [5]:

$$
\inf _{\substack{\rho(\cdot, 0)=f \\ \rho(\cdot, 1)=g}}\left\{\int_{0}^{1} \int_{M} \frac{P^{2}(x, t)}{\rho(x, t)} d \operatorname{vol}(x) d t \mid \partial_{t} \rho+\operatorname{div} P=0\right\} .
$$

Another characterization of the solution $\rho_{t}$ is as the shortest path joining $f$ to $g$, when the space of Borel probability measures on $M$ is metrized by 
the Wasserstein distance $d_{W}(f, g)$ whose square coincides with the infimum (14). Instead, we follow a Lagrangian approach yielding a third description of the same path. It begins with a Borel map $F: M \longrightarrow M$ pushing the mass of $f$ forward to $g$; indeed, among such maps, we choose the one which minimizes the average geodesic distance squared: $\int_{M} d^{2}(x, F(x)) f(x) d \operatorname{vol}(x)$. McCann showed this map to be unique [27], and to take the form $F(x)=$ $\exp _{x}(-\nabla \phi(x))$ where $\phi: M \longrightarrow \mathbf{R}$ is $d^{2} / 2$-concave, meaning, roughly speaking, $(\phi)^{c c}=\phi$ with the definitions $c(x, y):=d^{2}(x, y) / 2$ and

$$
\phi^{c}(y):=\inf _{x \in M} c(x, y)-\phi(x) .
$$

We introduce the parameter $t \in[0,1]$ to define a homotopy $F_{t}(x) \in$ $Z_{t}\left(x, F_{1}(x)\right)$ by

$$
F_{t}(x):=\exp _{x}(-t \nabla \phi(x))
$$

from the identity $F_{0}(x)=x$ to the map $F_{1}$. A Borel probability measure $\mu_{t}$ is then defined on $M$ by pushing the mass of $f$ forward through $F_{t}$, denoted $\mu_{t}:=\left(F_{t}\right)_{\#} \mu_{0}$ and meaning

$$
\int_{M} b(x) d \mu_{t}(x):=\int_{M} b\left(F_{t}(x)\right) f(x) d \operatorname{vol}(x)
$$

for every Borel test function $b$. After verifying that $\mu_{t}$ is absolutely continuous with respect to volume, we denote its density by $\rho_{t}(x):=d \mu_{t}(x) / d \operatorname{vol}(x)$. It remains to show that $\rho_{t}^{-1 / n}(z)$ dominates the greater side of (7) when $y=F_{1}(x)$. The proof relies on the optimality of $F_{1}$ in (16), which yields a pointwise estimate relating the Jacobian determinant $\mathrm{J}_{t}:=\operatorname{det} d F_{t}(x)$ to $J_{0}$ and $J_{1}$ via concavity of $\operatorname{det}^{1 / n}(\cdot)$. As this Jacobian includes a derivative of the exponential map, our argument naturally invokes the calculus of Jacobi fields developed, e.g., in Gallot, Hulin and Lafontaine [16] or Jost [22], to compute it along with the volume distortion (6).

Though the proof is conceptually simple, one needs to develop several tools of non-smooth analysis to cope with the lack of information on regularity of the map $F$. Unlike the Euclidean case, no regularity results are known for optimal transportation on manifolds. Therefore we generalize the approach of McCann [25,26] to identify the Jacobian of $F(x)$ almost everywhere and derive a non-smooth change of variables theory for optimal transport on manifolds. We conclude with a second application of this theory: demonstrating that convexity estimates satisfied by the family of densities $\rho_{t}$ on Euclidean space extend immediately to the Ricci non-negative setting. Introduced for measures on $\mathbf{R}^{n}$ under the name displacement convexity $[25,26]$, such inequalities were formally derived on Riemannian manifolds in recent work of Otto and Villani [28]. Theorem 6.2 is included below to justify some of their calculations.

Several applications of the Euclidean Prékopa-Leindler inequality can be generalized to the Riemannian setting via Corollary 1.2. For instance, as 
mentioned in [9] and [14], Maurey's property $(\tau)$ and its generalization by Bobkov and Ledoux [8] can be extended to Riemannian manifolds whose Ricci curvature has a strictly positive lower bound to recover the BakryEmery [3] logarithmic Sobolev inequality, as well as transport and concentration inequalities. This includes the following concentration of measure inequality, similar to the one obtained by Gromov and Milman [19], on a manifold $M$ verifying Ric $\geq R>0$ :

$$
\int e^{\lambda\left[f(x)-\int f d \sigma\right]} d \sigma(x) \leq e^{R^{-1} \lambda^{2}}
$$

where $f: M \rightarrow \mathbf{R}$ is 1 -Lipschitz, $\lambda \in \mathbf{R}$ and $d \sigma:=d \operatorname{vol} / \operatorname{vol}[M]$ is the normalized Riemannian volume measure.

Judging from Euclidean experience, we expect the nonsmooth differentiability and change of variables theory we establish for optimal maps to prove useful in many contexts. To begin, it shows these mappings represent each probability measure on a given manifold canonically as the (nearly diffeomorphic) image of an arbitrary reference measure. Furthermore, it will be seen that the geometry of the optimal map is intrinsically linked to the geometry of the underlying manifold, capturing detailed information about the curvature along geodesics relevant to the inequalities that we prove. Among other prospective applications of optimal transportation methods, one can mention Sobolev inequalities, isoperimetric theorems, logarithmic Sobolev inequalities (which imply rates of contraction for semigroup flows and dissipative dynamical systems) and the related displacement convexity discussed by Otto and Villani [28] for the entropy functional $H(\rho):=\int \rho \log \rho d \mathrm{vol}+\int \rho V d$ vol when $V: M \rightarrow \mathbf{R}$ satisfies Hess $V+$ Ric $\geq \lambda$. We imagine the differentiability, positivity, global optimality and injectivity results established below for interpolating maps to form the prerequisites for any subsequent study on the regularity of optimal maps.

The organization of the paper is as follows. Section 2 is devoted to recalling the relevant facts and terminology from Riemannian geometry, establishing the essential relationships between the exponential map, distance functions, cut locus, and the volume distortion factors $v_{t}(x, y)$. McCann's theorem on optimal mass transport is recalled in Sect. 3, where we explore some differential properties of $c$-concave functions. In particular, we prove they admit a second order Taylor expansion almost everywhere - a Hessian, in the sense of Aleksandrov [2] and Bangert [4]. Section 4 is devoted to the study of the differential of mass transport and to establishing a change of variables theorem for optimal maps. The penultimate Sect. 5 constructs the interpolation along mass transport and establishes optimality and injectivity properties which may hold independent interest. A final Sect. 6 gives the proof of our Main Theorem, its corollaries, and the displacement convexity result on Ricci nonnegative manifolds. The knowledgeable reader may prefer to begin consulting Sect. 6 directly for a conceptual and schematic 
outline of our main arguments, only then referring back to the intervening Sects. $2-5$ as necessary to fill in the missing concepts, definitions, and fine points of the proof.

This work represents part of the $\mathrm{PhD}$ thesis of the first named author at the Université de Marne-la-Vallée. He wishes to express deep gratitude to his advisor, Bernard Maurey, for providing ongoing guidance and support.

\section{Riemannian geometry: exponential map, distance functions, and volume distortion coefficients}

This section is devoted to recalling some aspects of Riemannian geometry, and using them to establish key lemmas required later. It begins with a discussion of the exponential map and Riemannian distance function, before relating the volume distortion factors $v_{t}(x, y)$ to derivatives of the exponential map. These factors are bounded by comparison with spaces of constant curvature in Corollary 2.2. The next lemma establishes crucial positivity properties for derivatives of the Riemannian distance function $d_{y}(\cdot):=d(\cdot, y)$, while a final proposition characterizes the cut locus of $y$ as the set of points where second differentiability fails for $d_{y}$. This characterization proves vital, for it implies that optimal transport never moves positive mass from its initial location all the way to the cut locus: the resulting degeneracies are confined to a set of measure zero.

For $x \in M$, the cut locus refers to the set $\operatorname{cut}(x) \subset M$ of all $z \in M$ which cannot be linked to $x$ by an extendable minimizing geodesic, meaning $z \notin$ $\cup_{t \in(0,1)} Z_{t}(x, M)$. The exponential map $\exp _{x}: T_{x} M \rightarrow M$ is differentiable at any tangent vector $v \in T_{x} M$ satisfying $\exp _{x} v \notin \operatorname{cut}(x)$ [16, §3.77], and its differential $Y_{x, v}:=d\left(\exp _{x}\right)_{v}$ gives a linear bijection between the tangent spaces $T_{x} M$ and $T_{\exp _{x} v} M$. We adopt the notation $Y:=Y_{x, v}$, suppressing the dependence on $x$ and on $v$. For any $y \in M$ denote by $d_{y}(\cdot):=d(\cdot, y)$ the distance function to $y$. The relationship between this distance and the exponential map is summarized by the formula $d_{y}\left(\exp _{y} v\right)=|v|$, which holds for any $v$ from the star-shaped domain around $0 \in T_{y} M$ which does not intersect $\left(\exp _{y}\right)^{-1}[\operatorname{cut}(y)]$. This shows the exponential map generates the minimal geodesics through $y$, and that the function $d_{y}^{2} / 2$ is smooth around any $x \notin \operatorname{cut}(y)$. Where $d_{y}^{2} / 2$ is differentiable, its gradient is related to the exponential map by the formula [27, Proposition 6],

$$
y=\exp _{x}\left[-\nabla d_{y}^{2}(x) / 2\right]
$$

and for $x \notin \operatorname{cut}(y)$ its Hessian $H=\operatorname{Hess}_{x} d_{y}^{2} / 2$ can be viewed either as a symmetric quadratic form on $T_{x} M$, or - more frequently - as a selfadjoint operator $H: T_{x} M \longrightarrow T_{x} M$. Note that (18) requires the existence of a minimal geodesic linking $x$ to $y$, and it is for this reason that completeness of the manifold is required. 
The coefficients $v_{t}(x, y)$ and $v_{1-t}(y, x)$ are linked to the differential of the exponential map and the Hessian of the distance function by the following lemma:

Lemma 2.1 (Volume distortion coefficients) Fix $x, y \in M$ with $x \notin$ $\operatorname{cut}(y)$ and let $\gamma(t):=\exp _{x}(t v)$ be the minimal geodesic joining $x=\gamma(0)$ to $y=\gamma(1)$. For $t \in[0,1]$ we introduce $Y(t):=d\left(\exp _{x}\right)_{t v}$ and $H(t):=$ $\operatorname{Hess}_{x} d_{\gamma(t)}^{2} / 2$. Then definition (6) implies

$$
v_{t}(x, y)=\frac{\operatorname{det} Y(t)}{\operatorname{det} Y(1)}=\operatorname{det} Y(t) Y(1)^{-1}>0
$$

and for $t \neq 1$

$$
v_{1-t}(y, x)=\operatorname{det} \frac{Y(t)(H(t)-t H(1))}{1-t} .
$$

Here the determinants are computed in Riemannian normal coordinates on each tangent space, or equivalently by expressing the coordinates of $Y(t)$ and $H(t)$ with respect to an orthonormal moving frame which has been parallel transported along $\gamma$.

Proof. Equality (19) follows easily from the definition of $v_{t}(x, y)$. The map $G_{t}(u):=\exp _{x}(t u)$ is a local diffeomorphism between a neighborhood of $v$ and a neighborhood of $\gamma(t)$; by the chain rule its differential at $v$ is $d\left(G_{t}\right)_{v}=t Y(t)$. Introduce the set $C_{r}:=\left\{u \in T_{x} M \mid \exp _{x} u \in B_{r}(y)\right\}$, which shrinks nicely to $v$ as $r$ tends to 0 , in the terminology of Rudin (c.f. Claim 4.5 below). Since geodesics through $x \in M$ correspond to straight lines through $0 \in T_{x} M$ under the exponential map, $G_{t}$ maps $C_{r}$ onto $Z_{t}\left(x, B_{r}(y)\right)$ as long as $B_{r}(y)$ is disjoint from the cut locus $\operatorname{cut}(x)$. Thus we get

$$
\operatorname{det}(t Y(t))=\operatorname{det} d\left(G_{t}\right)_{v}= \pm \lim _{r \rightarrow 0} \frac{\operatorname{vol}\left[Z_{t}\left(x, B_{r}(y)\right)\right]}{\operatorname{vol}\left[C_{r}\right]} .
$$

The sign must positive since $\operatorname{det} Y(t) \neq 0$ for any $t \in[0,1]$ and $Y(0)=I$. Using this equality at $t=1$, since $Z_{1}\left(x, B_{r}(y)\right)=B_{r}(y)$,

$0 \neq \frac{\operatorname{det} Y(t)}{\operatorname{det} Y(1)}=\lim _{r \rightarrow 0} \frac{\operatorname{vol}\left[Z_{t}\left(x, B_{r}(y)\right)\right]}{t^{n} \operatorname{vol}\left[B_{r}(y)\right]}=\lim _{r \rightarrow 0} \frac{\operatorname{vol}\left[Z_{t}\left(x, B_{r}(y)\right)\right]}{\operatorname{vol}\left[B_{t r}(y)\right]}=: v_{t}(x, y)$.

The second equality (20) follows from the first after characterizing $v_{t}(x, y)$ as the determinant of a matrix of Jacobi fields. Let us therefore say a few words about Jacobi fields. Let $x, y \in M$ and $\gamma(t):=\exp _{x} t v$ be a minimal geodesic joining $x=\gamma(0)$ to $y=\gamma(1)$. A Jacobi field is formally defined as a vector field $V$ along $\gamma$ satisfying the linear differential equation $V^{\prime \prime}(t)+R(V(t), \dot{\gamma}(t)) \dot{\gamma}(t)=0$. Here the derivatives of vector fields are the covariant derivatives along $\gamma, R$ is the Riemann curvature tensor on $M$, and $\dot{\gamma}(t)$ the tangent vector to $\gamma$ at $t$. In particular a Jacobi field along 
$\gamma$ is uniquely determined by the initial conditions $V(0)$ and $V^{\prime}(0)$. When there exists a non-zero Jacobi field along $\gamma$ vanishing at endpoints, one says that $x$ and $y$ are conjugate points. If $x$ and $y$ are conjugate points then $y \in \operatorname{cut}(x)$ (as in [16, §3.78]). When $x$ and $y$ are not conjugate points, a Jacobi field $V$ along $\gamma$ is characterized by $V(0)$ and $V(1)$. An equivalent and more intuitive way of defining Jacobi fields is to consider variations of $\gamma$ by geodesics. Consider a smooth family $\gamma_{s}$ of geodesic curves, which includes the geodesic of interest $\gamma_{0}:=\gamma$. Then the vector field $V(t):=\left.\frac{\partial}{\partial s}\right|_{s=0} \gamma_{s}(t)$ which generates this variation is a Jacobi field along $\gamma$. Conversely, every Jacobi field along $\gamma$ can be obtained in this way.

Returning to the proof of the lemma: because $\exp _{x}$ generates all geodesics through $x$, it is a well known fact that the matrix $t Y(t)$ is a matrix consisting of Jacobi fields along $\gamma$ [16]. (More precisely, $t Y(t) u$ is a Jacobi field along $\gamma$ whenever $u \in T_{x} M$.) Thus the linear combination $t Y(t) Y(1)^{-1}$ also forms a matrix of Jacobi fields along $\gamma$ which vanishes at $\gamma(0)$ and coincides with the identity matrix $I$ at $\gamma(1)$. We then have the following reformulation of (19): if $A(t)$ is the unique matrix of Jacobi fields along $\gamma$ satisfying $A(0)=0$ and $A(1)=I$, then

$$
v_{t}(x, y):=\operatorname{det} \frac{A(t)}{t} .
$$

Therefore if $B(t)$ is the unique matrix of Jacobi fields along $\gamma$ satisfying $B(0)=I$ and $B(1)=0$, then

$$
v_{1-t}(y, x)=\operatorname{det} \frac{B(t)}{1-t} .
$$

To establish (20) it is therefore sufficient to show that the matrix $B(t)=$ $Y(t)(H(t)-t H(1))$ is the required matrix of Jacobi fields. Fixing $w \in T_{x} M$, we will prove that $Y(t)(H(t)-t H(1)) w$ is a Jacobi field along $\gamma$. Recall $\gamma(t)=\exp _{x}(t v)$ and denote by $c(s)$ any curve such that $c(0)=x$ and $\dot{c}(0)=w\left(\right.$ such as $\left.c(s)=\exp _{x}(s w)\right)$. Now consider the following variation of $\gamma$ :

$$
\gamma_{s}(t):=\exp _{c(s)}\left(-t \nabla d_{y}^{2}(c(s)) / 2\right)
$$

The field of variation $V(t):=\left.\frac{\partial}{\partial s}\right|_{s=0} \gamma_{s}(t)$ is a Jacobi field along $\gamma$ since all the curves $\gamma_{s}$ are geodesics. Since (18) implies $\gamma_{s}(1)=y$ while $\gamma_{s}(0)=c(s)$, we have $V(1)=0$ and $V(0)=w$. We write

$$
\gamma_{s}(t)=\exp _{c(s)}\left(-\nabla d_{\gamma(t)}^{2}(c(s)) / 2+\nabla h(c(s))\right),
$$

where $h(z):=d_{\gamma(t)}^{2}(z) / 2-t d_{y}^{2}(z) / 2$. Invoking (18) again shows the function

$$
s \longrightarrow \exp _{c(s)}\left(-\nabla d_{\gamma(t)}^{2}(c(s)) / 2\right)=\gamma(t)
$$


is independent of $s$. At $s=0, c(0)=x, \nabla h(x)=0$ and $\nabla d_{\gamma(t)}^{2}(x) / 2=t v$. Thus differentiating (22) at $s=0$, the chain rule combines with (23) to yield

$$
\begin{aligned}
V(t) & =\left(d\left(\exp _{x}\right)_{t v} \operatorname{Hess}_{x} h\right) c^{\prime}(0) \\
& =Y(t)(H(t)-t H(1)) w .
\end{aligned}
$$

This shows that $Y(t)(H(t)-t H(1)) w$ is a Jacobi field. Moreover $B(t)=$ $Y(t)(H(t)-t H(1))$ satisfies the desired boundary conditions: $V(0)=w=$ $B(0) w$ and $V(1)=0$. Thus (20) follows from (21) and the lemma is established.

The previous characterization of $v_{t}$ together with the Bishop's comparison theorem leads to an estimate of $v_{t}$ in terms of Ricci curvature.

Corollary 2.2 (Comparison bound on volume distortion) Assume that Ric $\geq(n-1) k$ throughout $M$ for some $k \in \mathbf{R}$. Then for $x, y \in M$ with $y \notin \operatorname{cut}(x)$ and $t \in(0,1)$,

$$
v_{t}(x, y) \geq\left(\frac{S_{k}(t d(x, y))}{S_{k}(d(x, y))}\right)^{n-1},
$$

where $S_{k}$ is from equation (10). Equality holds when $M$ has constant sectional curvature equal to $k$.

Proof. Let $\gamma(t):=\exp _{x} t v$ be the minimal geodesic joining $x=\gamma(0)$ to $y=\gamma(1)$ from the lemma. Minimality implies this geodesic is free from conjugate points; it is parameterized by arc length rescaled by $|v|=$ $d(x, y)$. Thus the hypothesized Ricci bound yields $\operatorname{Ric}_{\gamma(t)}\left(\gamma^{\prime}(t), \gamma^{\prime}(t)\right) \geq$ $(n-1) k|v|^{2}$. Denoting the Jacobian determinant of the exponential map by $J_{n-1}(t):=d\left(\exp _{x}\right)_{t} v=\operatorname{det} Y(t)$, Theorem 15 of Bishop and Crittenden [7, $\S 11.10]$ asserts that

$$
t \longrightarrow(\operatorname{det} Y(t))\left(S_{k}(t|v|)\right)^{-(n-1)}
$$

is a non-increasing function of $t \in(0,1]$. From (19) this yields

$$
v_{t}(x, y)=\frac{\operatorname{det} Y(t)}{\operatorname{det} Y(1)} \geq\left(\frac{S_{k}(t|v|)}{S_{k}(|v|)}\right)^{n-1} .
$$

If $M$ has constant sectional curvature, a direct computation of the Jacobi fields making up $Y(t)$ yields equality in (24), thus concluding the corollary.

The following lemma is an elementary consequence of the triangle inequality which has a critical role to play in our proof. It provides half of the positivity we eventually require to deduce the Jacobian inequality (74) from the domination of means in Lemma 6.1. Note the relevance of the Hessian $H(t)-t H(1)$ to the distortion coefficient (20). 
Lemma 2.3 (Hessian positivity relating distance functions) Let $\gamma(t)=$ $\exp _{x}(t v)$ be the minimal geodesic linking $x \in M$ to $\gamma(1) \notin \operatorname{cut}(x)$. The self-adjoint operator $H(t)-t H(1)$ defined on $T_{x} M$ by $H(t)=\mathrm{Hess}_{x} d_{\gamma(t)}^{2} / 2$ has non-negative eigenvalues.

Proof. We begin by recording the following claim which will also prove useful later:

Claim 2.4 Let $x, y, z \in M$ with $z \in Z_{t}(x, y)$ for some $t \in(0,1)$. Then for all $m \in M$,

$$
t d^{2}(m, y) \leq d^{2}(m, z)+t(1-t) d^{2}(x, y) .
$$

This inequality is sharp, in the sense that choosing $m=x$ produces a case of equality.

Proof of claim. The triangle inequality combines with domination of the arithmetic by the geometric mean to yield, for any $\varepsilon>0$,

$$
\begin{aligned}
d^{2}(m, y) & \leq d^{2}(m, z)+d^{2}(z, y)+2 d(m, z) d(z, y) \\
& \leq\left(1+\varepsilon^{-1}\right) d^{2}(m, z)+(1+\varepsilon) d^{2}(z, y) .
\end{aligned}
$$

Choosing $\varepsilon=t /(1-t)$ and noting $d(z, y)=(1-t) d(x, y)$ establishes the inequality (25). To see $m=x$ produces a case of equality, invoke the relation $d_{z}(m)=t d_{y}(m)$ which follows from $z \in Z_{t}(m, y)$.

End of the proof of the lemma. Since $\gamma(t) \in Z_{t}(x, y)$, re-expressing the claim yields

$$
\alpha(m):=d_{\gamma(t)}^{2}(m) / 2+t(1-t) d_{y}^{2}(x) / 2-t d_{y}^{2}(m) / 2 \geq 0
$$

and attains the minimum value $0=\alpha(x)$. Since neither $y$ and nor $z=\gamma(t)$ belongs to $\operatorname{cut}(x)$, differentiating twice yields Hess $x \geq 0$. This coincides with the desired inequality.

In the sequel, it proves useful to characterize the cut locus $\operatorname{cut}(y)$ as the set of points where the distance function $d_{y}^{2} / 2$ must fail to be smooth. As mentioned above, $d_{y}^{2} / 2$ is as smooth as the manifold away from the cut locus of $y$. But $\operatorname{cut}(y)$ consists of two kinds of points: (i) those connected to $y$ by multiple minimizing geodesics, and (ii) those which are conjugate to $y$ but do not fall into class (i). The point of the following proposition is that differentiability of $d_{y}^{2}$ must fail - at first order in case (i), and at second order in case (ii). Moreover, the failure occurs with a definite sign: the Hessian diverges to $-\infty$ while remaining bounded above, according to Lemma 3.12.

Proposition 2.5 (Distances fail to be semiconvex at the cut locus) At each $x \in \operatorname{cut}(y)$, the square distance $\psi:=d_{y}^{2} / 2$ satisfies:

$$
\inf _{0<|v|<1} \frac{\psi\left(\exp _{x} v\right)+\psi\left(\exp _{x}-v\right)-2 \psi(x)}{|v|^{2}}=-\infty .
$$


Proof. For $x \in \operatorname{cut}(y)$ there are only two possibilities (see e.g. [16, §3.78]).

Case 1: There exist two distinct minimal geodesics joining $x$ and $y$. This is the easy case. If $\gamma(t)=\exp _{x}(t u)$ is a minimal geodesic joining $x=\gamma(0)$ to $y=\gamma(1)$, then by [27, Proposition 6] $-\dot{\gamma}(0)=-u$ is a supergradient of $\psi=d_{y}^{2} / 2$ at $x$ in the sense that for $v \rightarrow 0$ in $T_{x} M$,

$$
\psi\left(\exp _{x} v\right) \leq \psi(x)+\langle-u, v\rangle+o(|v|) .
$$

Of course we may write an equality in (27) when $\psi$ is differentiable. But precisely if there exists two minimal geodesics joining $x$ to $y$, the function $\psi$ is not differentiable at $x$ and the existence of two distinct supergradients satisfying (27) immediately implies that the infimum (26) diverges to $-\infty$.

Case 2: Only one minimal geodesic joins $x$ to $y$, in which case $x$ and $y$ are conjugate points, meaning some non-zero normal Jacobi field along this geodesic vanishes at both endpoints. This case is more tricky and requires the use of the second variation of energy formula. Our proof is directly inspired by the characterization of the cut locus given in Gallot, Hulin and Lafontaine [16, §3.73]. Assume the result of the lemma is false: i.e. there exists a constant $C>0$ such that

$$
\liminf _{v \rightarrow 0} \frac{\psi\left(\exp _{x} v\right)+\psi\left(\exp _{x}-v\right)-2 \psi(x)}{|v|^{2}} \geq-C .
$$

Let $\gamma(t)=\exp _{x}(t u)$ be the minimal geodesic joining $x=\gamma(0)$ to $y=\gamma(1)$. Recall (see Jost [22, p 169]) that the index form $I$ is a symmetric bilinear form defined on the space of all vector fields $X_{1}$ and $X_{2}$ along $\gamma$ by

$$
\begin{aligned}
I\left(X_{1}, X_{2}\right):= & \int_{0}^{1}\left(\left\langle X_{1}^{\prime}(t), X_{2}^{\prime}(t)\right\rangle-\left\langle R\left(\dot{\gamma}(t), X_{2}(t)\right) X_{1}(t), \dot{\gamma}(t)\right\rangle\right) d t \\
= & -\int_{0}^{1}\left\langle X_{1}(t), X_{2}^{\prime \prime}(t)+R\left(X_{2}(t), \dot{\gamma}(t)\right) \dot{\gamma}(t)\right\rangle d t \\
& +\left[\left\langle X_{1}(t), X_{2}^{\prime}(t)\right\rangle\right]_{t=0}^{t=1} .
\end{aligned}
$$

Here $X(t) \in T_{\gamma(t)} M$ while $X^{\prime}(t)$ denotes its covariant derivative along $\gamma$; the second identity follows from an integration by parts and the symmetries of the Riemann tensor.

Let $Y(t)$ be a non-zero normal Jacobi field along $\gamma$ vanishing at 0 and 1 . By scaling the overall size of the manifold and the vector field independently, it costs no generality to normalize the length of the geodesic so that $d(x, y)=$ $|u|=1$, and take $v:=Y^{\prime}(0)$ - which is necessarily non-zero - to be a unit vector. Let $Z_{1}$ be a parallel vector field along $\gamma$ with $Z_{1}(0)=Y^{\prime}(0)=v$ and let $Z(t):=(1-t) Z_{1}(t)$. Fix $\alpha>0$ small enough that

$$
-\frac{2}{\alpha}+I(Z, Z)<-C
$$


and for this $\alpha$ consider

$$
Y_{\alpha}(t):=Y(t)+\alpha Z(t) .
$$

The field $Y_{\alpha}$ is normal along $\gamma$. It satisfies $Y_{\alpha}(0)=\alpha v$ and $Y_{\alpha}(1)=0$.

We introduce the following variation of the geodesic $\gamma$ : for $s$ close to 0 ,

$$
\gamma_{s}(t):=f(s, t):=\exp _{\gamma(t)}\left(s Y_{\alpha}(t)\right)
$$

The energy of the curve $\gamma_{s}$ is by definition $E(s):=\frac{1}{2} \int_{0}^{1}\left|\partial_{t} \gamma_{s}(t)\right|^{2} d t$. The curve $\gamma_{s}$ joins the point $\exp _{x}(s \alpha v)$ to $y$. The definition of geodesic distance combined with Hölder's inequality gives

$$
\psi\left(\exp _{x} s \alpha v\right)=\frac{1}{2} d^{2}\left(\exp _{x}(s \alpha v), y\right) \leq \frac{1}{2}\left(\int_{0}^{1}\left|\partial_{t} \gamma_{s}(t)\right| d t\right)^{2} \leq E(s) .
$$

Note that for $s=0$, since $\gamma$ is a minimizing geodesic, there is equality in (30). So our assumption (28) gives:

$$
\liminf _{s \rightarrow 0} \frac{E(s)+E(-s)-2 E(0)}{|\alpha|^{2} s^{2}} \geq-C .
$$

On the other hand, we can compute the second derivative of $E$ with the second variation of energy formula (see e.g. Jost [22, p 164-165]),

$$
\begin{aligned}
E^{\prime \prime}(0)=- & \int_{0}^{1}\left\langle Y_{\alpha}(t), Y_{\alpha}^{\prime \prime}(t)+R\left(Y_{\alpha}(t), \dot{\gamma}(t)\right) \dot{\gamma}(t)\right\rangle \\
& +\left[\left\langle D_{s} \frac{\partial f}{\partial s}(s, t), \dot{\gamma}(t)\right\rangle\right]_{(s, t)=(0,0)}^{(s, t)=(0,1)}+\left[\left\langle Y_{\alpha}(t), Y_{\alpha}^{\prime}(t)\right\rangle\right]_{t=0}^{t=1} .
\end{aligned}
$$

Here $D_{s} \frac{\partial f}{\partial s}(s, t)$ is the covariant derivative of $\frac{\partial f}{\partial s}(s, t)$ with respect to $s$. Since $s \rightarrow f(s, t)$ is a geodesic (for fixed $t$ ) we have $D_{s} \frac{\partial f}{\partial s}(s, t)=0$. Thus

$$
\begin{aligned}
E^{\prime \prime}(0) & =I\left(Y_{\alpha}, Y_{\alpha}\right) \\
& =I(Y, Y)+2 \alpha I(Z, Y)+\alpha^{2} I(Z, Z) .
\end{aligned}
$$

Observe that $I(Y, Y)=0$ since $Y$ is a Jacobi field vanishing at endpoints and $I(Z, Y)=\left[\left\langle Z(t), Y^{\prime}(t)\right\rangle\right]_{t=0}^{t=1}=-|v|^{2}=-1$. Thus $E^{\prime \prime}(0)=-2 \alpha+$ $\alpha^{2} I(Z, Z)$ in equation (31), which contradicts (29), concluding the proof of the proposition. 


\section{Background on mass transport and properties of $c$-concave functions}

\subsection{Mass transport and c-concave functions}

This section recalls the theory of optimal mass transportation developed in the Riemannian context by McCann [27]. We fix the cost function $c(x, y):=d^{2}(x, y) / 2=d_{y}^{2}(x) / 2$ throughout. To begin, we recall a generalized Legendre transformation adapted to this choice of cost.

Definition 3.1 ( $c$-transforms and the subset $\mathcal{I}^{c}(X, Y)$ of $c$-concave functions) Let $X$ and $Y$ be two compact subsets of $M$. The set $I^{c}(X, Y)$ of c-concave functions (relative to $X$ and $Y$ ) is the set of functions $\phi$ : $X \rightarrow \mathbf{R} \cup\{-\infty\}$ not identically $-\infty$, for which there exists a function $\psi: Y \rightarrow \mathbf{R} \cup\{-\infty\}$ such that

$$
\phi(x)=\inf _{y \in Y} c(x, y)-\psi(y) \quad \forall x \in X .
$$

We refer to $\phi$ as the c-transform of $\psi$ and abbreviate (33) by writing $\phi=\psi^{c}$. Similarly, given $\phi \in \mathcal{I}^{c}(X, Y)$, we define its $c$-transform $\phi^{c} \in \mathcal{I}^{c}(Y, X)$ by

$$
\phi^{c}(y):=\inf _{x \in X} c(x, y)-\phi(x) \quad \forall y \in Y .
$$

We hope no confusion results from the tacit dependence of these transformations on the domain of the function being transformed. For $\phi \in$ $I^{c}(X, Y)$, it follows easily from (34) as in Rachev and Rüschendorf [31, $\S 3.3$ ] that

$$
\phi(x)=\inf _{y \in Y} c(x, y)-\phi^{c}(y) \quad \forall x \in X,
$$

which we abbreviate by writing $\phi^{c c}=\phi$, suppressing the domains of definition once more. As in [27], Lipschitz continuity of $\phi^{c}$ follows merely from compactness of $X$ and the locally Lipschitzian character of $c(x, y)$, whether or not $\phi: X \longrightarrow \mathbf{R} \cup\{-\infty\}$ is continuous. Thus it costs no generality to assume $\psi$ and $\phi$ are continuous and real-valued in definition (33), in view of (35).

For two Radon measures $\mu$ and $v$ on $M$, we say a map $T: M \longrightarrow M$ defined $\mu$-a.e. pushes $\mu$ forward to $v$ (or transports $\mu$ onto $v$ ) if $v$ is the image measure of $\mu$ under $T$, denoted $v=T_{\#} \mu$ where $\left(T_{\#} \mu\right)(B):=\mu\left(T^{-1}(B)\right)$ for all Borel sets $B \subset M$. The definition of the push-forward $T_{\#} \mu$ can equally well be expressed by asserting

$$
\int b(y) d\left(T_{\#} \mu\right)(y)=\int b(T(x)) d \mu(x)
$$

for all Borel functions $b: M \rightarrow \mathbf{R}_{+}$. We state now the central result of McCann [27, §5] on optimal mass transport. 
Theorem 3.2 (Optimal mass transport on manifolds [27]) Let $M$ be a complete, continuously curved Riemannian manifold. Fix two Borel probability measures $\mu \ll$ vol and $v$ on $M$, and two compact subsets $X$ and $Y \subset M$ containing the support of $\mu$ and $\nu$, respectively. Then there exists $\phi \in \mathcal{I}^{c}(X, Y)$ such that the map

$$
F(x):=\exp _{x}(-\nabla \phi(x))
$$

pushes $\mu$ forward to $v$. This map is uniquely characterized among all maps pushing $\mu$ forward $v$ by formula (37) with $\phi \in \mathcal{I}^{c}(X, Y)$. Furthermore $F$ is the unique minimizer of the quadratic cost $\int d^{2}(x, G(x)) d \mu(x)$ among all Borel maps $G: M \rightarrow M$ pushing $\mu$ forward to $v$ (apart from variations on sets of $\mu$-measure zero).

The map $F$ may be referred to either as the optimal map or optimal mass transport between $\mu$ and $\nu$.

Let us also recall one of the basic lemmas from its proof, which illuminates the structure of the map $F$. Given two compact subsets $X$ and $Y \subset M$ with $\phi \in \mathcal{I}^{c}(X, Y)$, one sees every $(x, y) \in X \times Y$ satisfy

$$
c(x, y)-\phi(x)-\phi^{c}(y) \geq 0,
$$

with equality when $\phi(x)=\inf _{y^{\prime} \in Y} c\left(x, y^{\prime}\right)-\phi^{c}\left(y^{\prime}\right)=c(x, y)-\phi^{c}(y)$.

Notation: Throughout the sequel it will be convenient to use the notation $\mathcal{X} \subset \subset$ to denote an open subset $\mathcal{X}$ of $M$ whose closure $\bar{X}$ is compact.

Lemma 3.3 (Elementary properties of $c$-concave functions [27]) Fix $\mathcal{X} \subset \subset M$ open and $Y \subset M$ compact. For $\phi \in \mathcal{I}^{c}(\bar{X}, Y)$ define $F(x):=$ $\exp _{x}(-\nabla \phi(x))$.

(a) The function $\phi$ is Lipschitz on $\bar{X}$ and hence differentiable almost everywhere on $\mathcal{X}$.

(b) Fix any point $x \in \mathcal{X}$ where $\phi$ is differentiable. Then $y=F(x)$ if and only if y minimizes (38) among $y^{\prime} \in Y$. In the latter case one has $\nabla \phi(x)=\nabla d_{y}^{2}(x) / 2$.

Proof. Detailed proofs can be found in [27, Lemmas 2 and 7]; (a) was discussed above and is rather standard, but for completeness we sketch here a proof of $(b)$. Let $y \in Y$ be a minimizer for (38), meaning $\phi^{c}(y)=$ $c(x, y)-\phi(x)$, and let $d_{y}$ denote, as always, the distance function to $y$. Again by (38)

$$
d_{y}^{2}(z) / 2-\phi(z)-\phi^{c}(y) \geq 0
$$

for every $z \in X$ with a minimum value of zero when $z=x$. Differentiation of (39) at $z=x$ yields

$$
\nabla \phi(x)=\nabla d_{y}^{2}(x) / 2 .
$$

Thus (18) yields $y=\exp _{x}\left(-\nabla d_{y}^{2}(x) / 2\right)=\exp _{x}(-\nabla \phi(x))=F(x)$. 
Conversely let $y:=F(x)$. Since $Y$ is compact there exists $y^{\prime} \in Y$ minimizing (38). By the previous computations $F(x)=y^{\prime}=y$ proving that $y$ minimizes (38).

Remark 3.4 (A priori Hessian bound for smooth c-concave functions) In the previous proof, when $\phi$ is twice differentiable at $x \notin \operatorname{cut}(F(x))$, computing second derivatives at the minimum $z=x$ of (39) yields

$$
\operatorname{Hess}_{x}\left(d_{y}^{2} / 2-\phi\right) \geq 0
$$

for $y=F(x)$. Heuristically, this provides the second bit of positivity we ultimately require to establish the Jacobian concavity estimate (74). In the next subsection, we introduce a non-smooth notion of Hessian which respects the above inequality.

A $c$-concave function is not necessarily differentiable everywhere and thus the mass transport map $F$ is not defined everywhere. However for fixed $x \in X$, the Lipschitz continuity of $\phi^{c}$ asserted in Lemma 3.3 on the compact set $Y$ guarantees that some $y \in Y$ minimizes (38). This motivates the definition below, which provides a (possibly multivalued) extension of the map $F(x)=\exp _{x}(-\nabla \phi(x))$ to all of $X$.

Definition 3.5 ( $c$-superdifferential $\partial^{c} \phi$ ) Let $X, Y$ be two compact sets of $M$. For $\phi \in \mathcal{I}^{c}(X, Y)$ and $x \in X$, the $c$-superdifferential of $\phi$ at $x$ is the non-empty set

$$
\begin{aligned}
\partial^{c} \phi(x) & :=\left\{y \in Y \mid \phi(x)+\phi^{c}(y)=c(x, y)\right\} \\
& =\{y \in Y \mid \phi(z) \leq \phi(x)+c(z, y)-c(x, y) \quad \forall z \in X\} .
\end{aligned}
$$

Example 3.6 (Multivalued extension) If $\phi \in \mathcal{I}^{c}(\bar{X}, Y)$ is differentiable at $x \in \mathcal{X} \subset \subset M$, then $\partial^{c} \phi(x)=\{F(x)\}=\left\{\exp _{x}(-\nabla \phi(x))\right\}$ according to Lemma $3.3(b)$.

\subsection{Semi-concavity and Hessians for c-concave functions}

We next recall some tools of non-smooth analysis from the work of Bangert [4] on topological manifolds, which allow us to handle the fact that $c$-concave functions are not twice differentiable in the usual sense. Since Bangert's topological definitions are independent of coordinate choices, it is convenient for us to frame our definitions in the Riemannian normal coordinate charts given by the exponential map.

A function $\phi: \Omega \rightarrow \mathbf{R}$ defined on an open subset $\Omega$ of $M$ is said to be superdifferentiable at $x \in \Omega$ with supergradient $v \in T_{x} M$ if for $u \rightarrow 0 \in T_{x} M$,

$$
\phi\left(\exp _{x} u\right) \leq \phi(x)+\langle v, u\rangle+o(|u|)
$$


The superdifferential of $\phi$ at $x$ refers to the convex set $\partial \phi(x) \subset T_{x} M$ of all supergradients at $x$.

A typical example of a function which admits supergradients everywhere is the square distance $d_{y}^{2} / 2$ to $y \in M$. Indeed, if $\gamma$ is a minimal geodesic joining $x=\gamma(0)$ to $y=\gamma(1)$, then $-\dot{\gamma}(0) \in \partial\left(d_{y}^{2} / 2\right)(x)$ was established in [27]. The following lemma combines with Definition 3.5 to extend this property to $c$-concave functions:

Lemma 3.7 (c-supergradients imply supergradients) Fix $\mathcal{X} \subset \subset M$ open, $Y \subset M$ compact, and $\phi \in \mathcal{I}^{c}(\overline{\mathcal{X}}, Y)$. Let $(x, y) \in \mathcal{X} \times Y$ and $v \in T_{x} M$ satisfy $|v|=d(x, y)$ and $\exp _{x}(-v)=y$. If $y \in \partial^{c} \phi(x)$ then $v \in \partial \phi(x)$.

Proof. Fix $(x, y) \in \mathcal{X} \times Y$ such that $y \in \partial^{c} \phi(x)$. For every $z \in \mathcal{X}$, (41) implies

$$
\phi(z) \leq \phi(x)+c(z, y)-c(x, y) .
$$

Any shortest vector $v \in T_{x} M$ satisfying $\exp _{x}(-v)=y$ has length $|v|=$ $d(x, y)$. Now [27, Proposition 6] generalizes (18) by asserting $v \in \partial\left(d_{y}^{2} / 2\right)(x)$. Thus for $z=\exp _{x} u$,

$$
c\left(\exp _{x} u, y\right)-c(x, y) \leq\langle v, u\rangle+o(|u|)
$$

as $u \rightarrow 0$. Combining the two inequalities yields the desired result (42).

The two previous examples are representatives of a much more general class of functions admitting non-empty superdifferentials. Designated by $-\phi \in \mathcal{F}(\Omega)$ in the notation of Bangert, we next introduce the semi-concave functions. First recall that a geodesic ball $B_{r}(x)$ of radius $r$ around $x \in M$ is said to be embedded if the exponential map $\exp _{x}: \tilde{B}_{r}^{x}(0) \rightarrow B_{r}(x)$ defines a diffeomorphism from the open ball $\tilde{B}_{r}^{x}(0) \subset T_{x} M$ onto $B_{r}(x) \subset M$. A geodesic ball $B_{r}(x)$ around $x$ is a convex embedded ball if it is embedded and geodesically convex - meaning every pair of points $y, z \in B_{r}(x)$ are joined by a unique geodesic of length less than $2 r$, and this geodesic is contained in $B_{r}(x)$. Small enough balls are always convex embedded balls according to [16, §2.90].

Definition 3.8 (Semi-concavity) Fix $\Omega \subset$ M open. A function $\phi: \Omega \rightarrow \mathbf{R}$ is semi-concave at $x_{0} \in \Omega$ if there exists a convex embedded ball $B_{r}\left(x_{0}\right)$ and a smooth function $V: B_{r}\left(x_{0}\right) \rightarrow \mathbf{R}$ such that $\phi+V$ is geodesically concave throughout $B_{r}\left(x_{0}\right)$. The function $\phi$ is semi-concave on $\Omega$ if it is semi-concave at each point of $\Omega$.

It is well-known that concave (and thus semi-concave) functions at $x \in M$ admit non-empty superdifferentials at all points near $x$. This allows the following definition of the Hessian, as a derivative of supergradients (equivalent to the normal coordinate multifunctions of $[4, \S 4.1]$ ): 
Definition 3.9 (Hessian) Let $\phi: \Omega \rightarrow \mathbf{R}$ be semi-concave on an open set $\Omega \subset M$. We say that $\phi$ has a Hessian $H$ at $x \in \Omega$ if $\phi$ is differentiable at $x$ and there exists a self-adjoint operator $H: T_{x} M \rightarrow T_{x} M$ satisfying

$$
\sup _{v \in \partial \phi\left(\exp _{x} u\right)}\left|\Pi_{x, u} v-\nabla \phi(x)-H u\right|=o(|u|)
$$

as $u \rightarrow 0$ in $T_{x} M$. Here $\Pi_{x, u}: T_{\exp _{x} u} M \rightarrow T_{x} M$ denotes parallel translation to $x$ along $\gamma(t):=\exp _{x}(t u)$. The Hessian of $\phi$ at $x$ may also be denoted by $\operatorname{Hess}_{x} \phi:=H$.

This definition coincides with the usual one for smooth functions. A more intuitive understanding of the Hessian follows from the fact that existence of a Hessian $H$ at $x$ for $\phi$ implies a second order Taylor expansion for $\phi$ around $x:$ as $u \rightarrow 0 \in T_{x} M$,

$$
\phi\left(\exp _{x} u\right)=\phi(x)+\langle\nabla \phi(x), u\rangle+\frac{1}{2}\langle H u, u\rangle+o\left(|u|^{2}\right) .
$$

It is remarkable that the converse also holds true: if $\psi$ is semi-concave around $x$ then (43) follows from (44). Even more remarkable is the following theorem, proved by Aleksandrov [2] in the Euclidean case and on manifolds by Bangert [4, §4.4]. (For modern proofs in $\mathbf{R}^{n}$ see [1] or [6].)

Theorem 3.10 (Aleksandrov-Bangert [4]) Let $\phi: \Omega \rightarrow M$ be semiconcave function on an open set $\Omega \subset M$. Then $\phi$ admits a Hessian almost everywhere on $\Omega$.

The observation enabling us to exploit this theory is that any $c$-concave function is semi-concave. This is proved in the next proposition, after establishing uniform semi-concavity locally for the squared distance functions $d_{y}^{2}$ as a special case. We require the following observation:

Lemma 3.11 (Local characterization of semi-concavity) Let $\phi: \Omega \rightarrow \mathbf{R}$ be a continuous function and fix $x_{0} \in \Omega$. Assume that there exists a neighborhood $U$ of $x_{0}$ and a positive constant $C$ such that for every $x \in U$ and $u \in T_{x} M$ one has,

$$
\limsup _{r \rightarrow 0} \frac{\phi\left(\exp _{x} r u\right)+\phi\left(\exp _{x}-r u\right)-2 \phi(x)}{r^{2}} \leq C .
$$

Then $\phi$ is semi-concave around $x_{0}$.

Proof. The function $h:=d_{x_{0}}^{2}$ is smooth around $x_{0}$ and has Hessian $2 I$ at $x_{0}$. So there exists a neighborhood $V$ of $x_{0}$ such that $\operatorname{Hess}_{x} h>I$ for every $x \in V$. Set $\psi:=C h-\phi$ and take a convex embedded ball $B \subset U \cap V$ centered at $x_{0}$. By construction every $x \in B$ and $u \in T_{x} M$ satisfy

$$
\liminf _{r \rightarrow 0} \frac{\psi\left(\exp _{x} r u\right)+\psi\left(\exp _{x}-r u\right)-2 \psi(x)}{r^{2}}>0 .
$$


We will prove that $\psi$ is geodesically convex on $B$. Let $\gamma:[0,1] \longrightarrow M$ be a geodesic contained in $B$ and set $f(t)=\psi(\gamma(t))$. The function $f$ : $[0,1] \longrightarrow \mathbf{R}$ is continuous. Applying (45) to $x=\gamma(t)$ with $u=\dot{\gamma}(t)$ we get

$$
\liminf _{r \rightarrow 0} \frac{f(t+r)+f(t-r)-2 f(t)}{r^{2}}>0
$$

for every $t \in(0,1)$. But this implies convexity of $f$ : indeed, for $t_{0}, t_{1} \in[0,1]$ and $s \in(0,1)$ we shall prove $f\left((1-s) t_{0}+s t_{1}\right) \leq(1-s) f\left(t_{0}\right)+s f\left(t_{1}\right)$. By subtracting an affine function, it costs no generality to assume that $f\left(t_{0}\right)=f\left(t_{1}\right)=0$. This does not affect inequality (46), and we need only argue that $f$ is non-positive on $\left(t_{0}, t_{1}\right)$. To derive a contradiction, suppose the continuous function $f$ assumes a positive maximum $f(t)>0$ at $t \in\left(t_{0}, t_{1}\right)$. Maximality ensures that for $r$ small enough one has

$$
f(t+r)+f(t-r)-2 f(t) \leq 0
$$

which contradicts (46). Thus $f$ is convex on $[0,1]$ and $\psi=C h-\phi$ is geodesically convex throughout $B$.

Lemma 3.12 (Hessian bound for distance squared) Fix $x, y \in M$ and a minimal geodesic $\gamma$ joining $x$ to $y$. Suppose $-k<0$ is a lower bound for the sectional curvatures at every point of $\gamma$. Setting $L(s):=s(\tanh s)^{-1}$, each $u \in T_{x} M$ satisfies

$$
\limsup _{r \rightarrow 0} \frac{d_{y}^{2}\left(\exp _{x} r u\right)+d_{y}^{2}\left(\exp _{x}-r u\right)-2 d_{y}^{2}(x)}{r^{2}} \leq 2 L(\sqrt{k} d(x, y)) .
$$

Proof. This is direct consequence of the second variation formula. Indeed assume $\gamma$ is parametrized by arc length, with $|\dot{\gamma}(t)|=1$ and joins the point $x=\gamma(0)$ to $y=\gamma(\ell)$. Introduce the parallel transport $u(t)$ of $u \in T_{x} M$ along $\gamma$ and the vector field

$$
X(t):=\alpha(t) u(t) \quad \text { where } \alpha(t):=\frac{\sinh (\sqrt{k}(\ell-t))}{\sinh (\sqrt{k} \ell)} .
$$

The vector field $X$ satisfies $X(0)=u$ and $X(\ell)=0$. Introduce the following variation of the geodesic $\gamma$ :

$$
\gamma_{r}(t):=f(r, t):=\exp _{\gamma(t)}(r X(t))
$$

and note that $\gamma_{r}(0)=\exp _{x}(r u)$ and $\gamma_{r}(\ell)=y$. The definition of the geodesic distance combined with Hölder's inequality gives

$$
d_{y}^{2}\left(\exp _{x} r u\right) \leq\left(\int_{0}^{\ell}\left|\partial_{t} \gamma_{r}(t)\right| d t\right)^{2} \leq \ell \int_{0}^{\ell}\left|\partial_{t} \gamma_{r}(t)\right|^{2} d t=: 2 \ell E\left(\gamma_{r}\right)
$$


For $r=0$ there is equality in (47) since $\gamma_{0}=\gamma$ is a geodesic. Thus we have,

$$
\frac{d_{y}^{2}\left(\exp _{x} r u\right)+d_{y}^{2}\left(\exp _{x}-r u\right)-2 d_{y}^{2}(x)}{r^{2}} \leq 2 \ell \frac{E\left(\gamma_{r}\right)+E\left(\gamma_{-r}\right)-2 E\left(\gamma_{0}\right)}{r^{2}} \text {. }
$$

The right hand side has a limit when $r \rightarrow 0$ given by the second variation formula (32), in which one boundary term vanishes since $r \longrightarrow f(r, t)$ is a geodesic for each $t$ :

$$
\begin{aligned}
\left.\frac{d^{2} E\left(\gamma_{r}\right)}{d r^{2}}\right|_{r=0} & =\int_{0}^{\ell}\left[\left|X^{\prime}(t)\right|^{2}-R(X(t), \dot{\gamma}(t), X(t), \dot{\gamma}(t))\right] d t \\
& =\int_{0}^{\ell}\left[\left(\alpha^{\prime}(t)\right)^{2}-(\alpha(t))^{2} R(u(t), \dot{\gamma}(t), u(t), \dot{\gamma}(t))\right] d t
\end{aligned}
$$

where $X^{\prime}$ is the covariant derivative of $X$ along $\gamma$ and $R$ is the Riemann tensor. Using the sectional curvature bound along $\gamma$ we obtain

$$
\begin{aligned}
\left.\frac{d^{2} E\left(\gamma_{r}\right)}{d r^{2}}\right|_{r=0} & \leq \int_{0}^{\ell}\left[\left(\alpha^{\prime}\right)^{2}+k \alpha^{2}\right] d t \\
& =(\sinh (\sqrt{k} \ell))^{-2} k \int_{0}^{\ell} \cosh (2 \sqrt{k}(t-\ell)) d t \\
& =(\sinh (\sqrt{k} \ell))^{-2} \frac{\sqrt{k}}{2} \sinh (2 \sqrt{k} \ell) \\
& =\frac{\sqrt{k} \cosh (\sqrt{k} \ell)}{\sinh (\sqrt{k} \ell)}
\end{aligned}
$$

Combined with (48) this proves the lemma.

Corollary 3.13 (Uniform semi-concavity of distance squared) Let $X$, $Y \subset M$ be compact. There exists a constant $C>0$ such that every $(x, y) \in$ $X \times Y$ and $u \in T_{x} M$ satisfy

$$
\limsup _{r \rightarrow 0} \frac{d_{y}^{2}\left(\exp _{x} r u\right)+d_{y}^{2}\left(\exp _{x}-r u\right)-2 d_{y}^{2}(x)}{r^{2}} \leq C .
$$

Proof. Every $x \in X$ and $y \in Y$ is linked by a minimal geodesic since the manifold $M$ is complete. The union $U$ of all such minimal geodesic segments starting in $X$ and ending in $Y$ is a closed bounded set, by compactness of $X$ and $Y$. Thus one can find a uniform lower bound $-k<0$ for sectional curvatures on $U$. Inequality (49) follows from the previous lemma since the diameter of $X \cup Y$ is finite.

Proposition 3.14 (c-concave functions are semi-concave) Fix $\mathcal{X} \subset \subset M$ open and $Y \subset M$ compact. A c-concave function $\phi \in I^{c}(\bar{X}, Y)$ is semiconcave on $\mathcal{X}$ (and hence admits a Hessian (43) almost everywhere in $\mathcal{X}$ ). 
Proof. For $x \in \mathcal{X}$, Definition 3.5 provides $y \in \partial^{c} \phi(x) \subset Y$. Every $\pm u \in$ $T_{x} M$ satisfies (41):

$$
\phi\left(\exp _{x} u\right) \leq \phi(x)+d_{y}^{2}\left(\exp _{x} u\right) / 2-d_{y}^{2}(x) / 2 .
$$

Corollary 3.13 now yields $C>0$ such that every $x \in \mathcal{X}$ and $u \in T_{x} M$ satisfies

$$
\limsup _{r \rightarrow 0} \frac{\phi\left(\exp _{x} r u\right)-\phi\left(\exp _{x}-r u\right)-2 \phi(x)}{r^{2}} \leq C .
$$

By Lemma 3.11 this implies $\phi$ is semi-concave around each point of $\mathcal{X}$. Aleksandrov and Bangert's Theorem 3.10 provides a Hessian almost everywhere.

\section{The optimal transport Jacobian}

Given two absolutely continuous probability measures $\mu, v \ll$ vol with respect to the Riemannian volume on $M$, denote their respective densities by $f(x)=d \mu(x) / d \operatorname{vol}(x)$ and $g(y)=d \nu(y) / d \operatorname{vol}(y)$. The goal of this section is to show that the optimal transport map $F: M \longrightarrow M$ pushing $\mu$ forward to $v$ in Theorem 3.2 is differentiable almost everywhere (in a sense made precise below) and its Jacobian determinant satisfies the equation

$$
f(x)=g(F(x)) \operatorname{det} d F_{x} .
$$

The form $F(x)=\exp _{x}(-\nabla \phi(x))$ of the optimal map reduces this to a Monge-Ampère equation in the Euclidean case. Our goal is to specify a precise sense in which equation (50) holds $f$ almost everywhere. As a byproduct, we derive a change of variables theorem for the (not necessarily Lipschitz) map $F: M \longrightarrow M$.

\subsection{Non-smooth differentiation of mass transport}

Example 3.6 demonstrates that the $c$-superdifferential $\partial^{c} \phi \subset X \times Y$ of $\phi \in \mathcal{I}^{c}(X, Y)$ provides a multivalued extension (40) of the map $F(x):=$ $\exp _{x}(-\nabla \phi(x))$ to points $x \in X$ where $\phi$ is not differentiable. The next proposition uses $\partial^{c} \phi$ to define a differential $d F_{x}$ for such optimal maps $F(x)$. From the chain rule for smooth functions, it is clear that $d F_{x}$ should involve the derivative of the exponential map and the Hessian of $\phi$.

Proposition 4.1 (Differentiating optimal transport) Fix $\mathcal{X} \subset \subset M$ be open and $Y \subset M$ compact. Let $\phi \in \mathcal{I}^{c}(\bar{X}, Y)$ and set $F(z):=\exp _{z}(-\nabla \phi(z))$. Fix a point $x \in \mathcal{X}$ where $\phi$ admits a Hessian (43). Then:

(a) $y:=F(x) \notin \operatorname{cut}(x)$ and setting $H:=\operatorname{Hess}_{x} d_{y}^{2} / 2$, one has $H-\operatorname{Hess}_{x} \phi$ $\geq 0$. 
(b) Introduce $Y:=d\left(\exp _{x}\right)_{-\nabla \phi(x)}$ and define $d F_{x}: T_{x} M \longrightarrow T_{y} M$ by $d F_{x}:=Y\left(H-\operatorname{Hess}_{x} \phi\right)$. Then as $u \rightarrow 0$ in $T_{x} M$,

$$
\sup _{\substack{\exp _{y} v \in \partial^{c} \phi\left(\exp _{x} u\right) \\|v|=d\left(y, \exp _{y} v\right)}}\left|v-d F_{x}(u)\right|=o(|u|) .
$$

Proof of (a). Suppose $\phi$ admits a Hessian (43) at $x \in \mathcal{X}$. Then $\phi$ is differentiable at $x$ and Example 3.6 shows that $\partial^{c} \phi(x)=\{F(x)\}=\{y\}$. Thus for every $z \in \mathcal{X}$, (41) yields

$$
\phi(z) \leq \phi(x)+d_{y}^{2}(z) / 2-d_{y}^{2}(x) / 2 .
$$

Taking $z=\exp _{x}( \pm u)$ and $\psi:=d_{y}^{2} / 2$ gives

$$
\frac{\phi\left(\exp _{x} u\right)+\phi\left(\exp _{x}-u\right)-2 \phi(x)}{|u|^{2}} \leq \frac{\psi\left(\exp _{x} u\right)+\psi\left(\exp _{x}-u\right)-2 \psi(x)}{|u|^{2}} .
$$

As $|u| \rightarrow 0$ the left hand side tends to $\left\langle\operatorname{Hess}_{x} \phi(u), u\right\rangle$ by hypothesis, so the right hand side is bounded below. Proposition 2.5 then ensures that $x \notin \operatorname{cut}(y)$, or equivalently $y \notin \operatorname{cut}(x)$.

From (52) we also observe that the function

$$
h(z):=d_{y}^{2}(z) / 2-\phi(z)
$$

has a minimum at $z=x$. The Taylor expansion (44) then implies the existence and non-negativity of its Hessian: $\operatorname{Hess}_{x} h=H-\operatorname{Hess}_{x} \phi \geq 0$. $\triangle$

Proof of $(b)$. Fix a unit tangent vector $u \in T_{x} M$ and set $x_{s}=\exp _{x}(s u)$. For $y_{s} \in \partial^{c} \phi\left(x_{s}\right)$ we want to establish the estimate $\left|v_{s}-d F_{x}(s u)\right|=o(s)$, where $v_{s} \in T_{y} M$ is the shortest vector such that $y_{s}=\exp _{y} v_{s}$, and the error term is independent of $u$.

Introduce $u_{s} \in T_{x_{s}} M$ such that $y_{s}=\exp _{x_{s}} u_{s}$ with $\left|u_{s}\right|=d\left(x_{s}, y_{s}\right)$ and let $w_{s}:=u_{s}+\nabla d_{y}^{2}\left(x_{s}\right) / 2$. Then

$$
y_{s}=\exp _{x_{s}}\left(-\nabla d_{y}^{2}\left(x_{s}\right) / 2+w_{s}\right) .
$$

Applying Lemma 3.7 to $y_{s} \in \partial^{c} \phi\left(x_{s}\right)$ yields $-u_{s} \in \partial \phi\left(x_{s}\right)$ and hence $w_{s} \in \partial h\left(x_{s}\right)$, where $h$ is as in (53). Recall that $h$ has a Hessian at $x$ satisfying $d F_{x}=Y \operatorname{Hess}_{x} h$, which by definition (43) means

$$
\Pi_{x, s u} w_{s}=s \operatorname{Hess}_{x} h(u)+o(s) .
$$

Thus the curve $w_{s}$ through $(x, 0) \in T M$ is differentiable at $s=0$ and we can identify the vertical component of its tangent vector as $\dot{w}_{s=0}=\operatorname{Hess}_{x} h(u)$. Recall from (18) that the function $z \rightarrow \exp _{z}\left(-\nabla d_{y}^{2}(z) / 2\right)=y$ is constant outside $\operatorname{cut}(y)$. Differentiating (54) using the chain rule then yields

$$
\begin{aligned}
\dot{y}_{s=0} & =d\left(\exp _{x_{0}}\right)_{-\nabla d_{y}^{2}\left(x_{0}\right) / 2}\left(\dot{w}_{0}\right) \\
& =Y \dot{w}_{0} \\
& =d F_{x}(u) .
\end{aligned}
$$


The appearance of $Y=d(\exp )_{-\nabla \phi(x)}$ in the second identity follows from $\nabla h\left(x_{0}\right)=0$ in (53). Finally, since $y_{0}=y$ we have

$$
\begin{aligned}
y_{s} & =\exp _{y}\left(s \dot{y}_{0}+o(s)\right) \\
& =\exp _{y}\left(s d F_{x}(u)+o(s)\right)=\exp _{y} v_{s} .
\end{aligned}
$$

The size of the error term $o(s)$ here does not depend on the unit vector $u$ since it did not depend on $u$ in (55). Comparison with (51) ends the proof of the proposition.

\subsection{Jacobian equation and optimal changes of variables}

Having established an almost everywhere notion for the differential $d F_{x}$ of an optimal map $F: M \longrightarrow M$, we now verify the Jacobian equation (50).

Theorem 4.2 (Jacobian identity a.e.) Let $\mu \ll$ vol and $v \ll$ vol be two compactly supported Borel probability measures and denote their $L^{1}(M, \mathrm{vol})$ densities by $f$ and $g$, respectively. Fix domains $\mathcal{X} \subset \subset$ and $y \subset \subset M$ containing the support of $\mu$ and $\nu$, respectively. Suppose $\phi \in \mathcal{I}^{c}(\bar{X}, \bar{y})$ induces $F: \mathcal{X} \longrightarrow \bar{y}$ defined by $F(x):=\exp _{x}(-\nabla \phi(x))$ which pushes $\mu$ forward to $v$. Then there exists a Borel set $K \subset \mathcal{X}$ of full measure for $\mu$ such that

(a) $\phi$ admits a Hessian $\operatorname{Hess}_{x} \phi$ at each $x \in K$, and hence $F(x) \notin \operatorname{cut}(x)$.

(b) For $x \in K$, setting $Y:=d\left(\exp _{x}\right)_{-\nabla \phi(x)}$ and $H:=\operatorname{Hess}_{x} d_{F(x)}^{2} / 2$, one has

$$
f(x)=g(F(x)) \operatorname{det}\left[Y\left(H-\operatorname{Hess}_{x} \phi\right)\right] \neq 0 .
$$

Proof. Recall that $\phi^{c} \in \mathcal{I}^{c}(\overline{\mathcal{y}}, \bar{X})$. Absolute continuity of both measures $\mu$ and $v$ ensures that $F^{*}(y):=\exp _{y}\left(-\nabla \phi^{c}(y)\right)$ is the optimal mass transport pushing $v$ forward to $\mu$, as in [27, Corollary 10]. The map $F^{*}$ is almost everywhere the inverse of $F$ since, for all $(x, y) \in \bar{X} \times \bar{y}$, we have the equivalence $y \in \partial^{c} \phi(x) \Leftrightarrow x \in \partial^{c} \phi^{c}(y)$.

Introduce the sets:

$$
\begin{aligned}
& E_{\phi}:=\left\{x \in \mathcal{X} \mid \operatorname{Hess}_{x} \phi \text { exists }\right\} \\
& E_{\phi^{c}}:=\left\{y \in \mathcal{Y} \mid \operatorname{Hess}_{y} \phi^{c} \text { exists }\right\} .
\end{aligned}
$$

Note that the map $F$ is well defined on $E_{\phi}$ and that for $x \in E_{\phi}$ Proposition 4.1(a) yields $F(x) \notin \operatorname{cut}(x)$ and $\operatorname{Hess}_{x}\left(d_{F(x)}^{2} / 2-\phi\right) \geq 0$. One can make the same observation for $\phi^{c}$ on $E_{\phi^{c}}$. Therefore introduce

$$
\begin{aligned}
\tilde{E}_{\phi} & :=\left\{x \in E_{\phi} \mid \operatorname{Hess}_{x}\left(d_{F(x)}^{2} / 2-\phi\right)>0\right\} \\
\tilde{E}_{\phi^{c}} & :=\left\{y \in E_{\phi^{c}} \mid \operatorname{Hess}_{y}\left(d_{F^{*}(y)}^{2} / 2-\phi^{c}\right)>0\right\} \\
\Omega & :=\left\{x \in \tilde{E}_{\phi} \mid F(x) \in \tilde{E}_{\phi^{c}}\right\} .
\end{aligned}
$$


For $x \in E_{\phi}$ we set, as before, $Y:=d\left(\exp _{x}\right)_{-\nabla \phi(x)}$ and $H:=\operatorname{Hess}_{x} \nabla d_{F(x)}^{2} / 2$ and

$$
d F_{x}:=Y\left(H-\operatorname{Hess}_{x} \phi\right) .
$$

For $x \in \tilde{E}_{\phi}$, the linear map $d F_{x}: T_{x} M \longrightarrow T_{F(x)} M$ is a bijection, since $Y$ is injective when $\exp _{x}(-\nabla \phi(x)) \notin \operatorname{cut}(x)$. For $y \in E_{\phi^{c}}$ we define $d F_{y}^{*}$ analogously. The proof of the theorem will be carried out in three steps:

Claim 4.3 (Inverse function theorem for optimal maps) Let $x \in E_{\phi}$ such that $F(x) \in E_{\phi^{c}}$. Then $(a) x \in \Omega$, (b) $\operatorname{det} d F_{x}>0$, and $(c) d F_{F(x)}^{*}=\left(d F_{x}\right)^{-1}$.

Claim 4.4 (Density of nice points) $\mu\left(\tilde{E}_{\phi}\right)=\mu(\Omega)=1$.

Claim 4.5 (Equivalence of algebraic and geometric Jacobians) Let $x \in \Omega$. Then $\partial^{c} \phi\left(B_{r}(x)\right)$ shrinks nicely to $y:=F(x)$ when $r \rightarrow 0$ and

$$
\lim _{r \rightarrow 0} \frac{\operatorname{vol}\left[\partial^{c} \phi\left(B_{r}(x)\right)\right]}{\operatorname{vol}\left[B_{r}(x)\right]}=\operatorname{det} d F_{x} .
$$

Here shrinks nicely means there exists $R(r) \rightarrow 0$ as $r \rightarrow 0$ such that $\partial^{c} \phi\left(B_{r}(x)\right) \subset B_{R(r)}(y)$ fills a non-zero volume fraction of $B_{R(r)}(y)$ in the limit; c.f. Rudin [32].

We postpone the proofs of these three claims until after the proof of the theorem. Setting

$$
K:=\left\{\begin{array}{l|l}
x \in \Omega & \begin{array}{c}
x \text { is a Lebesgue point of } f \text { where } f(x) \neq 0 \\
\text { and } F(x) \text { is a Lebesgue point of } g
\end{array}
\end{array}\right\},
$$

part (a) of the theorem follows from Proposition 4.1(a). Recall that $L^{1}(M, \mathrm{vol})$ functions have Lebesgue points vol-a.e. on $M$. Absolute continuity of measures $\mu$ and $\nu$ combines with Claim 4.4 and the definition of mass transport $F$ to give $\mu(K)=1$.

To address (b), let us fix $x \in K$. Since $x$ is a Lebesgue point of $f$ one has

$$
f(x)=\lim _{r \rightarrow 0} \frac{\mu\left[B_{r}(x)\right]}{\operatorname{vol}\left[B_{r}(x)\right]} .
$$

By Claim 4.5, $\partial^{c} \phi\left(B_{r}(x)\right)$ shrinks nicely to $F(x)$, so local differentiation of measures together with the fact that $F(x)$ is a Lebesgue point for $g$ implies

$$
\lim _{r \rightarrow 0} \frac{v\left[\partial^{c} \phi\left(B_{r}(x)\right)\right]}{\operatorname{vol}\left[\partial^{c} \phi\left(B_{r}(x)\right)\right]}=g(F(x)) .
$$

Observe that Example 3.6 yields $F^{-1}(V) \subset \mathcal{X} \cap \partial^{c} \phi^{c}(V)$, and the difference has zero volume since it consists only of points where the Lipschitz function $\phi$ fails to be differentiable. Absolute continuity of $\mu$ yields $\nu[V]=$ 
$\mu\left[F^{-1}(V)\right]=\mu\left[\partial^{c} \phi(V)\right]$ since $F$ pushes $\mu$ forward to $v$. A similar argument invoking absolute continuity of $v$ shows $\mu\left[B_{r}(x)\right]=v\left[\partial^{c} \phi\left(B_{r}(x)\right)\right]$ since $F^{*}: y \longrightarrow \bar{X}$ pushes $v$ forward to $\mu$. Thus (59) becomes

$$
\lim _{r \rightarrow 0} \frac{\mu\left[B_{r}(x)\right]}{\operatorname{vol}\left[\partial^{c} \phi\left(B_{r}(x)\right)\right]}=g(F(x)) .
$$

Claim 4.5 combined with (58) and (60) gives

$$
\begin{aligned}
f(x) & =\lim _{r \rightarrow 0} \frac{\mu\left[B_{r}(x)\right]}{\operatorname{vol}\left[B_{r}(x)\right]} \\
& =\lim _{r \rightarrow 0} \frac{\mu\left[B_{r}(x)\right]}{\operatorname{vol}\left[\partial^{c} \phi\left(B_{r}(x)\right)\right]} \frac{\operatorname{vol}\left[\partial^{c} \phi\left(B_{r}(x)\right)\right]}{\operatorname{vol}\left[B_{r}(x)\right]} \\
& =g(F(x)) \operatorname{det} d F_{x} .
\end{aligned}
$$

This completes the proof of the theorem.

Proof of Claim 4.3. We begin by observing the definition of $\Omega$ shows $\operatorname{det} d F_{x}$ has the same sign as det $Y$, which can be seen to be positive from (19) since $Y(0)=I$. Thus part $(b)$ of the claim will follow from part (a). Now fix $x \in E_{\phi}$ such that $y:=F(x) \in E_{\phi^{c}}$ and $u \in T_{x} M$. Let $x_{s}=\exp _{x}(s u)$ for $s \rightarrow 0$ and $y_{s} \in M$ such that $y_{s} \in \partial^{c} \phi\left(x_{s}\right)$. If $v_{s}$ is the smallest vector of $T_{y} M$ such that $y_{s}=\exp _{y} v_{s}$, Proposition 4.1(b) gives $v_{s}=s d F_{x}(u)+o(s)$. We can then apply the same argument for $\phi^{c}$ at $F(x)$ to get: $s u=d F_{F(x)}^{*} v_{s}+o(s)=s d F_{F(x)}^{*} d F_{x} u+o(s)$. Taking $s \rightarrow 0$ gives $d F_{F(x)}^{*} d F_{x} u=u$, which shows that $d F_{x}$ and $d F_{F(x)}^{*}$ are inverse to each other, so that $x \in \tilde{E}_{\phi}$ by (56). Similarly $F(x) \in \tilde{E}_{\phi^{c}}$ and so parts (c), (a) and hence (b) of the claim are all established.

Proof of Claim 4.4. Claim 4.3 gives $\Omega=\left\{x \in E_{\phi} \mid F(x) \in E_{\phi^{c}}\right\}$ and so

$$
\mu(\Omega)=\mu\left(E_{\phi} \cap F^{-1}\left(E_{\phi^{c}}\right)\right) .
$$

By definition of mass transport $\mu\left(F^{-1}\left(E_{\phi^{c}}\right)\right)=v\left(E_{\phi^{c}}\right)$. The result follows from the semi-concavity established in Proposition 3.14 and Theorem 3.10 of Aleksandrov and Bangert, which combine with absolute continuity of the measures $\mu$ and $v$ to ensure $\mu\left(E_{\phi}\right)=v\left(E_{\phi^{c}}\right)=1$.

Proof of Claim 4.5. Fix $x \in \Omega$ and set $y=F(x)$. For $z \in M$ let $\tilde{B}_{r}^{z}(0)$ again denote the ball in $T_{z} M$ of radius $r$ centered at the origin of $T_{z} M$. Set $c_{1}=\left\|d F_{x}\right\|$ and $c_{2}=\left\|\left(d F_{x}\right)^{-1}\right\|$. We first prove that for every $\varepsilon>0$ there exists $\delta>0$ such that for every $r<\delta$ one has

$\exp _{y}\left(\left(1+\varepsilon c_{1}\right)^{-1} d F_{x} \tilde{B}_{r}^{x}(0)\right) \subset \partial^{c} \phi\left(B_{r}(x)\right) \subset \exp _{y}\left(\left(1+\varepsilon c_{2}\right) d F_{x} \tilde{B}_{r}^{x}(0)\right)$.

Fix $\varepsilon>0$ and let $\delta>0$ such that Proposition 4.1(b) applies to $F$ at $x$ with an error term $o(u)$ less than $\varepsilon|u|$ whenever $|u|<\delta$. Fixing 
$r<\delta$, pick $u \in \tilde{B}_{r}^{x}(0)$ and suppose $\exp _{y} v \in \partial^{c} \phi\left(\exp _{x} u\right)$ with $|v|=$ $d\left(y, \exp _{y} v\right)$. By Proposition 4.1, $\left|v-d F_{x}(u)\right|_{y} \leq \varepsilon|u|_{x} \leq \varepsilon r$ showing $v \in d F_{x}\left(u+\varepsilon\left(d F_{x}\right)^{-1} \tilde{B}_{r}^{y}(0)\right) \subset\left(1+\varepsilon c_{2}\right) d F_{x}\left(\tilde{B}_{r}^{x}(0)\right)$. Since $u \in \tilde{B}_{r}^{x}(0)$ and $\exp _{y} v \in \partial^{c} \phi\left(\exp _{x} u\right)$ were arbitrary, exponentiation yields the second inclusion of (61):

$$
\partial^{c} \phi\left(B_{r}(x)\right) \subset \exp _{y}\left(\left(1+\varepsilon c_{2}\right) d F_{x}\left(\tilde{B}_{r}^{x}(0)\right)\right) .
$$

On the other hand, we may apply the same reasoning starting from $u \in\left(d F_{x}\right)^{-1} \tilde{B}_{r}^{y}(0)$ : as long as $c_{2} r<\delta$ then the shortest vector $v \in T_{y} M$ such that $\exp _{y} v \in \partial^{c} \phi\left(\exp _{x} u\right)$ satisfies $v \in d F_{x}(u)+\varepsilon c_{2} \tilde{B}_{r}^{y}(0) \subset\left(1+c_{2} \varepsilon\right) \tilde{B}_{r}^{y}(0)$, and exponentiation yields

$$
\partial^{c} \phi\left(\exp _{x}\left(\left(d F_{x}\right)^{-1} \tilde{B}_{r}^{y}(0)\right)\right) \subset \exp _{y}\left(1+\varepsilon c_{2}\right) \tilde{B}_{r}^{y}(0) .
$$

Applying the same argument to $\phi^{c}$ at $y=F(x)$ yields for small enough $r>0$,

$$
\partial^{c} \phi^{c}\left(\exp _{y}\left(d F_{x} \tilde{B}_{r}^{x}(0)\right)\right) \subset \exp _{x}\left(1+\varepsilon c_{1}\right) \tilde{B}_{r}^{x}(0),
$$

where $\left(d F_{y}^{*}\right)^{-1}=d F_{x}$ has been noted from Claim 4.3. Rescaling $r$ by a factor $\left(1+\varepsilon c_{1}\right)$ gives $\partial^{c} \phi^{c}\left(\exp _{y}\left(\left(1+\varepsilon c_{1}\right)^{-1} d F_{x} \tilde{B}_{r}^{x}(0)\right)\right) \subset \exp _{x}\left(\tilde{B}_{r}^{x}(0)\right)=$ $B_{x}(r)$. Since $A \subset \partial^{c} \phi\left(\partial^{c} \phi^{c}(A)\right)$, we get

$$
\exp _{y}\left(1+\varepsilon c_{1}\right)^{-1} d F_{x}\left(\tilde{B}_{r}^{x}(0)\right) \subset \partial^{c} \phi\left(B_{x}(r)\right) .
$$

Together with (62) this establishes (61). Note that (61) asserts that in the Riemannian normal coordinates around $y, \partial^{c} \phi\left(B_{r}(x)\right)$ will be contained in a scaled copy of a fixed ellipsoid, only slightly larger than one it contains; the scale factor $\left(1+\varepsilon c_{2}\right)\left(1+\varepsilon c_{1}\right)$ is as close to unity as we please for $r>0$ small enough. Thus $\partial^{c} \phi\left(B_{r}(x)\right)$ shrinks nicely to $y=F(x)$ and

$$
\lim _{r \rightarrow 0} \frac{\operatorname{vol}\left[\partial^{c} \phi\left(B_{r}(x)\right)\right]}{\operatorname{vol}\left[B_{r}(x)\right]}=\lim _{r \rightarrow 0} \frac{\left|d F_{x} \tilde{B}_{r}^{x}(0)\right|}{\left|\tilde{B}_{r}^{y}(0)\right|}= \pm \operatorname{det} d F_{x} .
$$

But det $d F_{x}>0$ by Claim 4.3, so the proof of (57) is complete.

Remark 4.6 (Improvements) Although not needed here, Claims 4.3 and 4.5 can be improved to yield stronger Inverse function and Jacobian theorems for optimal maps. For example, assume $\phi^{c}(y)$ differentiable at $F(x) \in y$ while $\phi$ admits a Hessian at $x \in \mathcal{X}$. Then $\phi^{c}(y)$ admits a Hessian at $F(x)$ if and only if $d F_{x}: T_{x} M \longrightarrow T_{F(x)} M$ is bijective. The proof is patterned closely after the Euclidean Theorems A.1-2 [26].

Similarly, for investigating displacement convexity in Sect. 6, it will be useful to record the follow change of variables theorem as a corollary to Theorem 4.2. 
Corollary 4.7 (Change of variables theorem for optimal maps) Choose $d \mu=f d \mathrm{vol}$ and $d v=g d \mathrm{vol}$ from Theorem 4.2, supported on domains $\mathcal{X} \subset \subset$ and $\mathcal{Y} \subset \subset M$, with a map $F(x):=\exp _{x}(-\nabla \phi(x))$ and $\phi \in$ $I^{c}(\overline{\mathcal{X}}, \bar{y})$ such that $v=F_{\#} \mu$. Define $K \subset \mathcal{X}$ with $\mu(K)=1$ and the Jacobian $\mathrm{J}(x):=\operatorname{det}\left[Y\left(H-\operatorname{Hess}_{x} \phi\right)\right]$ of $F$ at $x \in K$ as in the theorem. If $A(\rho)$ is a Borel function on $[0, \infty)$ with $A(0)=0$ then

$$
\int_{M} A(g(y)) d \operatorname{vol}(y)=\int_{K} A\left(\frac{f(x)}{\mathrm{J}(x)}\right) \mathrm{J}(x) d \operatorname{vol}(x) .
$$

(Either both integrals are undefined or both take the same value in $\overline{\mathbf{R}}$ ).

Proof. Using $d v=g d \mathrm{vol}, A(0)=0$, and the change of variables formula (36) defining $v=F_{\#} \mu$, we find

$$
\begin{aligned}
\int_{M} A(g(y)) d \operatorname{vol}(y) & =\int_{\{g>0\}} \frac{A(g(y))}{g(y)} d v(y) \\
& =\int_{M} 1_{\{g>0\}}(F(x)) \frac{A(g(F(x))}{g(F(x))} d \mu(x) \\
& =\int_{K \cap F^{-1}(\{g>0\})} A\left(\frac{f(x)}{\mathrm{J}(x)}\right) \frac{\mathrm{J}(x)}{f(x)} d \mu(x)
\end{aligned}
$$

where the last equality follows from the Jacobian identity proved in Theorem 4.2 on the set $K \subset \mathcal{X}$. Since $K \subset F^{-1}(\{g>0\})$ the corollary has been established.

\section{Optimal interpolating maps and densities}

The next subject of our attention will be the family of maps $F_{t}(x)=$ $\exp _{x}(-t \nabla \phi(x))$ which interpolate along geodesics from the identity map $x=F_{0}(x)$ to an optimal map $y=F_{1}(x)$. Ultimately, we shall want to extend the differentiability a.e. and Jacobian formulas proved for $t=1$ to the interval $t \in(0,1)$. This is accomplished using results of the previous section, after proving that the set of $c$-concave potentials is star-shaped around 0 , meaning $(t \phi)^{c c}=t \phi$ follows from $\phi^{c c}=\phi$. Theorem 3.2 then shows $F_{t}$ to be the optimal map from the measure $d \mu(x):=f(x) d \operatorname{vol}(x)$ to the image $\mu_{t}=\left(F_{t}\right)_{\#} \mu$ defined by equation (36). However, to invoke the results of the preceding section, it is also necessary to know $\mu_{t}$ is absolutely continuous with respect to volume, having a density $\rho_{t} \in L^{1}(M, \mathrm{vol})$ given by its Radon-Nikodym derivative $\rho_{t}(x)=d \mu_{t}(x) / d \operatorname{vol}(x)$. For this we need to quantify injectivity of $F_{t}: \mathcal{X} \longrightarrow M$. Establishing these facts are the goals of the present section. We begin with star-shapedness of the set of $c$-concave functions and injectivity of interpolant maps.

Lemma 5.1 (c-concave potentials form a star-shaped set) Fix $t \in[0,1]$ and compact sets $X \subset M$ and $Y \subset M$. Define $Z_{t}(X, Y)=\bigcup_{x \in X} Z_{t}(x, Y)$ similarly to (5). If $\phi \in \mathcal{I}^{c}(X, Y)$ then $t \phi \in \mathcal{I}^{c}\left(X, Z_{t}(X, Y)\right)$. 
Proof. The lemma is nearly as trivial for $t=0$ as for $t=1$ because $0 \in \mathcal{I}^{c}(X, X)$. Therefore, fix $t \in(0,1)$ and $y \in Y$. We begin by considering the special case $\phi(x):=c(x, y)=d^{2}(x, y) / 2-$ also denoted by $\phi:=d_{y}^{2} / 2$ - and establishing the representation

$t d_{y}^{2}(m) / 2=\inf _{z \in Z_{t}(X, y)}\left\{d_{z}^{2}(m) / 2+\inf _{\left\{x \in X \mid z \in Z_{t}(x, y)\right\}} t(1-t) d_{y}^{2}(x) / 2\right\} \quad \forall m \in X$

of $t \phi$ as a $c$-concave function in $\mathcal{I}^{c}\left(X, Z_{t}(X, y)\right) \subset \mathcal{I}^{c}\left(X, Z_{t}(X, Y)\right)$.

For any $m, x \in X$ and $z \in Z_{t}(x, y)$, the triangle inequality (25) yields

$$
t d_{y}^{2}(m) \leq d_{z}^{2}(m)+t(1-t) d_{y}^{2}(x) .
$$

Multiplying by $1 / 2$ shows $t \phi=t d_{y}^{2} / 2$ cannot exceed the double infimum (63). Claim 2.4 also asserts that $x=m$ produces equality in (64), thus proving (63).

Having established the claim for the special case $\phi:=d_{y}^{2} / 2$, we move on to the general case. Since $\phi=\phi^{c c}$ by (35), obviously

$$
t \phi(x)=\inf _{y \in Y} t c(x, y)-t \phi^{c}(y) .
$$

We have already shown that $t c(x, y)=t d_{y}^{2}(x)$ is in $\mathcal{I}^{c}\left(X, Z_{t}(X, Y)\right)$ for each $y \in Y$; as an infimum of such functions, $t \phi$ is also $c$-concave and in $\mathcal{I}^{c}\left(X, Z_{t}(X, Y)\right)$.

As an immediate corollary, Lemma 5.1 shows the interpolant map to be optimal in the mass transport setting.

Corollary 5.2 (Interpolant optimality) Fix a Borel probability measure $\mu \ll$ vol compactly supported in $X \subset \subset M$ and a compact set $Y \subset M$. Fix $\phi \in \mathcal{I}^{c}(\bar{X}, Y)$ and set $F_{t}(x):=\exp _{x}(-t \nabla \phi(x))$. For each $t \in[0,1]$, the map $F_{t}$ coincides with the optimal map pushing $\mu$ forward to the image measure $\mu_{t}=\left(F_{t}\right)_{\#} \mu$ defined by (36).

Proof. Setting $X:=\bar{X}$, Lemma 5.1 asserts $t \phi \in \mathcal{I}^{c}\left(X, Z_{t}(X, Y)\right)$ hence $c$-concave. Example 3.6 implies $F_{t}(x) \in Z_{t}(X, Y)$ for each $x \in X$ where $F_{t}(x)$ is defined - thus $\mu$-a.e. since $\mu \ll$ vol, in view of Rademacher's theorem and Lemma 3.3(a). The map $F_{t}$ pushes $\mu$ forward to $\mu_{t}$ by construction; McCann's Theorem 3.2 asserts the desired optimality, namely that $F_{t}$ minimizes average square distance transported among all maps pushing $\mu$ forward to $\mu_{t}$.

Lemma 5.3 (Interpolant injectivity) Let $\mathcal{X} \subset \subset M$ be open and $Y \subset M$ compact. Fix $\phi \in \mathcal{I}^{c}(\bar{X}, Y), t \in(0,1)$ and set $F_{t}(x):=\exp _{x}(-t \nabla \phi(x))$. If $F_{t}(x)=F_{t}\left(x^{\prime}\right)$ at two points $x, x^{\prime} \in \mathcal{X}$ of differentiability for $\phi$, then $x^{\prime}=x$. 
Proof. Assume $\phi$ is differentiable at two points $x, x^{\prime} \in \mathcal{X}$ having the same image $m:=F_{t}(x)=F_{t}\left(x^{\prime}\right)$ under $F_{t}$. Setting $y:=F_{1}(x)$ and $y^{\prime}:=F_{1}\left(x^{\prime}\right)$ implies $m \in Z_{t}(x, y) \cap Z_{t}\left(x^{\prime}, y^{\prime}\right)$.

Squaring and summing, the triangle inequalities

$$
\begin{aligned}
& d_{x}\left(y^{\prime}\right) \leq d_{x}(m)+d_{m}\left(y^{\prime}\right) \\
& d_{x^{\prime}}(y) \leq d_{x^{\prime}}(m)+d_{m}(y)
\end{aligned}
$$

yield

$$
\begin{aligned}
d_{x}^{2}\left(y^{\prime}\right)+d_{x^{\prime}}^{2}(y) \leq & d_{x}^{2}(m)+d_{m}^{2}(y)+d_{x^{\prime}}^{2}(m)+d_{m}^{2}\left(y^{\prime}\right)+2 d_{x} d_{y^{\prime}}(m) \\
& +2 d_{x^{\prime}} d_{y}(m) \\
= & d_{x}^{2}(y)+d_{x^{\prime}}^{2}\left(y^{\prime}\right) \\
& +2\left[d_{x} d_{y^{\prime}}+d_{x^{\prime}} d_{y}-d_{x} d_{y}-d_{x^{\prime}} d_{y^{\prime}}\right](m) \\
= & d_{x}^{2}(y)+d_{x^{\prime}}^{2}\left(y^{\prime}\right)-2 t(1-t)\left[d_{x}(y)-d_{x^{\prime}}\left(y^{\prime}\right)\right]^{2} \\
\leq & d_{x}^{2}(y)+d_{x^{\prime}}^{2}\left(y^{\prime}\right) .
\end{aligned}
$$

The last two equalities (67) and (68) follow from $d_{x}(y)=d_{x}(m) / t=$ $d_{y}(m) /(1-t)$ and similar expressions for $d_{x^{\prime}}\left(y^{\prime}\right)$. This inequality is in fact a Riemannian parallelogram inequality (draw a picture!).

On the other hand, Example 3.6 combines with the differentiability of $\phi$ to give $y \in \partial^{c} \phi(x)$ and $y^{\prime} \in \partial^{c} \phi\left(x^{\prime}\right)$. Smith and Knott's characterization of $\partial^{c} \phi$ using $c=d^{2} / 2$-cyclical monotonicity (see [17, Theorem 2.7] and references there) gives

$$
c\left(x, y^{\prime}\right)+c\left(x^{\prime}, y\right) \geq c(x, y)+c\left(x^{\prime}, y^{\prime}\right) .
$$

But this contradicts (69) unless equalities hold throughout (65-69). The first equality (65) forces the minimal geodesic from $x$ through $m$ to extend to $y^{\prime}$ as well as to $y$. On the other side of $m$, this same geodesic must pass through $x^{\prime}$ as well as $x$, if equality (66) is to be satisfied. So $m$ separates $x, x^{\prime}$ from $y, y^{\prime}$, all five points lying on a single geodesic. Finally $d_{x}(y)=d_{x^{\prime}}\left(y^{\prime}\right)$ from equality (69). Since $m$ divides both of these equal length segments in ratio $t:(1-t)$, we conclude $x=x^{\prime}$ and $y=y^{\prime}$.

The key property we shall require of $\mu_{t}$ is that it is given by a density $\rho_{t}=d \mu_{t} / d$ vol with respect to Riemannian volume. It is enough to verify:

Proposition 5.4 (Absolute continuity of the interpolant) Let $\mu \ll$ vol and $v \ll$ vol be absolutely continuous and compactly supported Borel probability measures on $M$. Fix open sets $\mathcal{X} \subset \subset M$ and $\mathcal{Y} \subset \subset M$ containing the support of $\mu$ and $\nu$, respectively, and choose $\phi \in \mathcal{I}^{c}(\overline{\mathcal{X}}, \bar{y})$ such that $F_{1}$ pushes $\mu$ forward to $\nu$, where $F_{t}(x):=\exp _{x}(-t \nabla \phi(x))$. For each $t \in(0,1)$ the image measure $\mu_{t}=\left(F_{t}\right)_{\#} \mu$ defined by (36) is absolutely continuous with respect to Riemannian volume. 
Proof. We reintroduce $E_{\phi}:=\left\{x \in \mathcal{X} \mid \operatorname{Hess}_{x} \phi\right.$ exists $\}$. The maps $F:=F_{1}$ and $F_{t}$ are well defined on $E_{\phi}$. For $x \in E_{\phi}$ we know by Proposition 4.1(a) that $F(x) \notin \operatorname{cut}(x)$ and so $F_{t}(x) \notin \operatorname{cut}(x)$. Introduce also

$$
\tilde{E}_{t}:=\left\{x \in E_{\phi} \mid \operatorname{Hess}_{x}\left(d_{F_{t}(x)}^{2} / 2-t \phi\right)>0\right\} .
$$

Claim $5.5 \mu\left(\tilde{E}_{t}\right)=1$.

Proof of the claim. By Lemma 2.3, $\operatorname{Hess}_{x}\left(d_{F_{t}(x)}^{2} / 2-t d_{F(x)}^{2} / 2\right) \geq 0$ and so

$$
\tilde{E}_{t} \supset \tilde{E}_{1} \text {. }
$$

Since the measures $\mu$ and $\nu$ are absolutely continuous with respect to Riemannian volume, Claim 4.4 can be applied and asserts $\tilde{E}_{\phi}:=\tilde{E}_{1}$ has full measure, so $1=\mu\left(\tilde{E}_{\phi}\right) \leq \mu\left(\tilde{E}_{t}\right)$.

We assume the set $\tilde{E}_{t}$ is $\sigma$-compact. If it was not, using the regularity of the measure $\mu$, one could always replace it by some $\sigma$-compact subset carrying the full measure of $\mu[32, \S 2.18]$.

We need to prove that for any Borel set $A$ satisfying $\operatorname{vol}(A)=0$ we have $\mu_{t}(A)=0$. By definition of mass transport and Claim 5.5 one has $\mu_{t}(A)=\mu\left(F_{t}^{-1}(A)\right)=\mu\left(F_{t}^{-1}(A) \cap \tilde{E}_{t}\right)$. Since $\mu$ is absolutely continuous, it is enough to show that

$$
\operatorname{vol}\left(F_{t}^{-1}(A) \cap \tilde{E}_{t}\right)=0 .
$$

In other words, it is enough to show that the image $\left(F_{t}\right)_{\#} \operatorname{vol}_{\tilde{E}_{t}}$ of the Riemannian volume measure on $\tilde{E}_{t}$ is absolutely continuous (with respect to Riemannian volume). Here $\mathrm{vol}_{B}$ denotes the restriction of the Riemannian measure to any Borel set $B \subset M$. We start with:

Claim 5.6 (Lipschitz control on inverse interpolant) Let $K$ be a compact subset of $\tilde{E}_{t}$. For every $x \in K$ there exists a constant $k_{x}>0$ such that for every $z \in K$,

$$
d\left(F_{t}(x), F_{t}(z)\right) \geq k_{x} d(x, z) .
$$

Consequently, the image of $\mathrm{vol}_{K}$ under $F_{t}$ is an absolutely continuous measure on $K^{\prime}:=F_{t}(K)$.

Proof of the claim. Lemma 5.3 shows $F_{t}: K \longrightarrow K^{\prime}$ is bijective. Lemma 5.1 combines with (51) to yield a first order Taylor expansion for $F_{t}$ at each $x \in K$, so $F_{t}$ is continuous on the compact set $K$. It follows that $F_{t}$ : $K \longrightarrow K^{\prime}:=F_{t}(K)$ is a homeomorphism. Our choice of $K \subset \tilde{E}_{t}$ combines with (19) to yield nonsingularity of the derivative $d\left(F_{t}\right)_{x}:=Y(t)(H(t)-$ $\left.t \operatorname{Hess}_{x} \phi\right)$ defined by $Y(t):=d\left(\exp _{x}\right)_{-t \nabla \phi(x)}$ and $H(t):=\operatorname{Hess}_{x} d_{F_{t}(x)}^{2} / 2$. Now assume the claim fails for some $x \in K$. Then there exist a sequence $x_{k}$ such that

$$
d\left(F_{t}(x), F_{t}\left(x_{k}\right)\right)<d\left(x, x_{k}\right) / k .
$$


We can find a subsequence of $\left(x_{k}\right)$, also denoted by $\left(x_{k}\right)$, that converges to some $z \in K$. By continuity and injectivity of $F_{t}, z=x$ and so $x_{k} \rightarrow x$. Let $u_{k} \in T_{x} M$ and $w_{k} \in T_{F_{t}(x)} M$ be the smallest vectors for which $x_{k}=\exp _{x} u_{k}$ and $F_{t}\left(x_{k}\right)=\exp _{F(x)} w_{k}$. By (72), $\left|w_{k}\right|=o\left(\left|u_{k}\right|\right)$.

Since $d\left(F_{t}\right)_{x}$ is invertible, there exists constants $c \leq C$ such that every $u \in T_{x} M$ satisfies

$$
c|u| \leq\left|d\left(F_{t}\right)_{x}(u)\right| \leq C|u| .
$$

Proposition 4.1(b) asserts $\left|w_{k}-d\left(F_{t}\right)_{x}\left(u_{k}\right)\right|=o\left(\left|u_{k}\right|\right)$ which implies that $\left|w_{k}\right|$ is of the same order than $\left|u_{k}\right|$, contradicting $\left|w_{k}\right|=o\left(\left|u_{k}\right|\right)$. The first part of the claim is therefore established.

Using standard arguments from measure theory, we shall now deduce that the image of $\operatorname{vol}_{K}$ under $F_{t}$ is absolutely continuous. The previous estimate (71) tells us that $K=\bigcup_{k} K_{k}$ where

$$
K_{k}=\left\{x \in K \mid \forall z \in K, d\left(F_{t}(x), F_{t}(z)\right) \geq \frac{1}{k} d(x, z)\right\} .
$$

Continuity of $F_{t}$ shows the sets $K_{k}$ to be closed, hence compact. But $F_{t}$ : $K_{k} \longrightarrow K_{k}^{\prime}:=F_{t}\left(K_{k}\right)$ has the property that $F_{t}^{-1}$ is Lipschitz. By a classical argument using the Vitali covering lemma, the Lipschitz map $F_{t}^{-1}$ cannot increase the volume of any subset of $K_{k}^{\prime}$ by factor greater than $k^{n}$. Thus the image of $\operatorname{vol}_{K_{k}}$ under $F_{t}: K_{k} \longrightarrow K_{k}^{\prime}$ is absolutely continuous. The image of $\mathrm{vol}_{K}$ under $F_{t}$ is an increasing limit of the images of vol $K_{k}$ under $F_{t}$ and so itself absolutely continuous, which concludes the proof of the claim. $\triangle$

End of the proof of the proposition. To deduce the absolute continuity of $\left(F_{t}\right)_{\#} \mathrm{Vol}_{\tilde{E}_{t}}$ from Claim 5.6, write $\tilde{E}_{t}=\bigcup K_{j}$ as an increasing union of compact sets $K_{j}$. The image of $\operatorname{vol}_{\tilde{E}_{t}}$ under $F_{t}$ is the increasing limit of the images of $K_{j}$ under $F_{t}$ which are absolutely continuous from the claim. Consequently the image of $\operatorname{vol}_{\tilde{E}_{t}}$ under $F_{t}$ is absolutely continuous. For $\operatorname{vol}(A)=0,(70)$ has now been verified. This implies the absolute continuity desired for $\left(F_{t}\right)_{\#} \mu$.

\section{Proof of Main Theorem and its corollaries}

We are finally in a position to pull together the technical ingredients developed in foregoing sections to establish our main objectives. The basic tool for proving our inequalities are the interpolating maps $F_{t}: M \longrightarrow M$ of Sect. 5, which allow us to localize geometrical inequalities under an integral and reduce them to matrix inequalities derived from domination of the geometric by the arithmetic mean. For such inequalities, non-negativity of the matrices is crucial. Such local inequalities capture geometrical aspects of the manifold through the appearance of volume distortion coefficients $v_{t}(x, y)$ in the concavity statement (74) relating the Jacobian determinant $\mathrm{J}_{t}(x)$ of the map $F_{t}$ at $x \in M$ to $\mathrm{J}_{0}(x)=1$ and $\mathrm{J}_{1}(x)$. 
Lemma 6.1 (Jacobian inequality) Fix $X \subset \subset M$ open, $Y \subset M$ compact, and a c-concave potential $\phi \in \mathcal{I}^{c}(\overline{\mathcal{X}}, Y)$. For each $t \in[0,1]$, define $F_{t}$ : $X \longrightarrow Z_{t}(\bar{X}, Y)$ by $F_{t}(z):=\exp _{z}(-t \nabla \phi(x))$. If $\phi$ admits a Hessian (43) at $x \in \mathcal{X}$, then $Y(t):=d\left(\exp _{x}\right)_{-t \nabla \phi(x)}$ and $H(t):=\operatorname{Hess}_{x} d_{F_{t}(x)}^{2} / 2$ both exist and the Jacobian determinant

$$
\mathrm{J}_{t}(x):=\operatorname{det} Y(t)\left(H(t)-t \operatorname{Hess}_{x} \phi\right)
$$

satisfies

$$
\mathrm{J}_{t}^{1 / n}(x) \geq(1-t)\left[v_{1-t}(F(x), x)\right]^{1 / n}+t\left[v_{t}(x, F(x))\right]^{1 / n} \mathrm{~J}_{1}^{1 / n}(x) .
$$

Note that Lemma 5.1 and Proposition 4.1 identify $\mathrm{J}_{t}(x)=\operatorname{det} d F_{t}(x)$ defined by (73) as the Jacobian determinant of the map $F_{t}: \mathcal{X} \longrightarrow Z_{t}(\bar{X}, Y)$.

Proof. Fix a point $x \in \mathcal{X}$ where $\phi$ admits a Hessian in the sense of Bangert and Aleksandrov (43). Proposition 4.1 asserts $F_{1}(x) \notin \operatorname{cut}(x)$ and Lemma 3.3 implies $d\left(x, F_{1}(x)\right)=|\nabla \phi(x)|$, so we conclude $F_{t}(x) \notin \operatorname{cut}(x)$ for $t \in[0,1]$. Thus $Y(t):=d\left(\exp _{x}\right)_{-t \nabla \phi(x)}$ and $H(t):=\operatorname{Hess}_{x} d_{F_{t}(x)}^{2} / 2$ are both well-defined.

Inequality (74) is trivial when $t=1$, so fix $t \in[0,1)$ and write

$$
\mathrm{J}_{t}(x)=\operatorname{det} Y(t) \operatorname{det}\left[(1-t) \frac{H(t)-t H(1)}{1-t}+t\left(H(1)-\operatorname{Hess}_{x} \phi\right)\right] .
$$

Non-negativity of the matrices $H(t)-t H(1)$ and $H(1)-\operatorname{Hess}_{x} \phi$ follow from the triangle inequality via Lemma 2.3 and $c$-concavity of $\phi$ via Proposition 4.1(a), respectively. Moreover, concavity of $\operatorname{det}^{1 / n}(\cdot)$ on the set of non-negative $n \times n$ real symmetric matrices is a well-known consequence of the domination of the geometric by the arithmetic mean. Combining $1 / n$-concavity of the determinant with (75) yields

$$
\begin{aligned}
\mathrm{J}_{t}^{1 / n}(x) \geq & \left(\operatorname{det}^{1 / n} Y(t)\right) \\
& \times\left((1-t) \operatorname{det}^{1 / n} \frac{H(t)-t H(1)}{1-t}+t \operatorname{det}^{1 / n}\left(H(1)-\operatorname{Hess}_{x} \phi\right)\right) \\
= & (1-t) \operatorname{det}^{1 / n} \frac{Y(t)(H(t)-t H(1))}{1-t}+t \operatorname{det}^{1 / n} Y(t)\left(H(1)-\operatorname{Hess}_{x} \phi\right) \\
= & (1-t) \operatorname{det}^{1 / n} \frac{Y(t)(H(t)-t H(1))}{1-t}+t \operatorname{det}^{1 / n} Y(t) Y(1)^{-1} \mathrm{~J}_{1}^{1 / n}(x) .
\end{aligned}
$$

The lemma follows from identification of the volume distortion factors (19-20) in Lemma 2.1, after noticing $t \longrightarrow F_{t}(x)$ is the minimal geodesic linking $x$ to $F(x)$. 
Proof of Main Theorem. Fix $f, g, h \geq 0$ on $M$ satisfying hypothesis (7). We first address the case in which the sets $A$ and $B$ carrying unit mass for $f$ and $g$ are both bounded subsets of $M$. Introduce absolutely continuous probability measures $\mu$ and $v$ whose densities are given by $f 1_{A}$ and $g 1_{B}$, respectively. Choose open sets $\mathcal{X}$ and $y$ such that $A \subset \subset X \subset \subset M$ and $B \subset \subset \mathcal{Y} \subset \subset M$, and set $X:=\bar{X}$ and $Y:=\bar{y}$. There exists $\phi \in \mathcal{I}^{c}(X, Y)$ such that $F(x):=\exp _{x}(-\nabla \phi(x))$ is the optimal mass transport pushing $\mu$ forward to $v$, according to Theorem 3.2. We introduce the interpolant map $F_{t}:=\exp _{x}(-t \nabla \phi(x))$ and the image $\mu_{t}=\left(F_{t}\right)_{\#} \mu$, which is given by a density $\rho_{t}:=d \mu_{t} / d \mathrm{vol}$ in view of Proposition 5.4.

Throughout the proof $t$ is fixed, but we may write $f=\rho_{0}, g=\rho_{1}$, and $F=F_{1}$. Since $t \phi \in \mathcal{I}^{c}\left(X, Z_{t}(X, Y)\right)$ by Lemma 5.1, Theorem 4.2 applies to both $F_{1}$ and $F_{t}$. It asserts the Jacobian identities

$$
\begin{aligned}
& f(x)=g(F(x)) \mathrm{J}_{1}(x) \neq 0 \\
& f(x)=\rho_{t}\left(F_{t}(x)\right) \mathrm{J}_{t}(x) \neq 0
\end{aligned}
$$

hold on a set $K \subset \mathcal{X}$ of measure 1 for $\mu$; for $x \in K, \phi$ admits a Hessian (43) and $\mathrm{J}_{t}(x)$ and $\mathrm{J}_{1}(x)$ are defined by (73). Applying Lemma 6.1 to (77) yields

$$
\frac{1}{\rho_{t}\left(F_{t}((x))^{1 / n}\right.} \geq(1-t)\left[\frac{v_{1-t}(F(x), x)}{f(x)}\right]^{1 / n}+t\left[v_{t}(x, F(x)) \frac{\mathrm{J}_{1}(x)}{f(x)}\right]^{1 / n} .
$$

Now the set $L:=K \cap A \cap F^{-1}(B)$ has full measure for $\mu$. For $x \in L$ we have $F_{t}(x) \in Z_{t}(x, F(x))$ and hypothesis (7) yields

$$
\frac{1}{h\left(F_{t}(x)\right)^{1 / n}} \leq(1-t)\left[\frac{v_{1-t}(F(x), x)}{f(x)}\right]^{1 / n}+t\left[\frac{v_{t}(x, F(x))}{g(F(x))}\right]^{1 / n} .
$$

Comparing (78-79) with (76) shows

$$
h\left(F_{t}(x)\right) \geq \rho_{t}\left(F_{t}(x)\right)
$$

for all $x \in L$. Regularity of the measure $\mu$ permits us to replace $L$ if necessary by a negligibly smaller set which is $\sigma$-compact. Continuity of $F_{t}$ on $K$ is implied by its differentiability there (51), thus $F_{t}(L)$ is $\sigma$ compact, hence Borel. Since $\left(F_{t}\right)_{\#} \mu_{0}=\mu_{t},(36)$ and (80) imply the desired conclusion:

$$
\begin{aligned}
\int_{F_{t}(L)} h(z) d \operatorname{vol}(z) & \geq \int_{M} 1_{F_{t}(L)} \rho_{t} d \mathrm{vol} \\
& =\int_{A} 1_{F_{t}(L)}\left(F_{t}(x)\right) f(x) d \operatorname{vol}(x) \geq \mu(L)=1 .
\end{aligned}
$$

Note if $\mu[A]=v[B]=\varepsilon>0$ instead of 1 , the conclusion from (7) would have been $\int_{M} h \geq \varepsilon$ instead of (81). 
We now turn to the case in which $A$ and $B \subset M$ are unbounded. In this case there exist bounded sets $A_{\varepsilon} \subset A$ and $B_{\varepsilon} \subset B$ chosen so that $\mu\left[A_{\varepsilon}\right]=v\left[B_{\varepsilon}\right]=1-\varepsilon$. If $h$ satisfies (7) for all $(x, y) \in A \times B$, it certainly satisfies it for all $(x, y) \in A_{\varepsilon} \times B_{\varepsilon}$. From the previous argument we conclude $\int_{M} h \geq 1-\varepsilon$. Since $\varepsilon>0$ was arbitrary the theorem has been established.

Proof of Corollary 1.1. Fix $p \geq-1 / n$ and $t \in[0,1]$. Let $f, g, h: M \longrightarrow$ $\mathbf{R}_{+}$be non-negative functions. First assume $f, g \in L^{1}(M$, vol $)$ and $p>$ $-1 / n$. Also assume $\|f\|_{1}$ and $\|g\|_{1}$ are non-zero; otherwise the conclusion is a trivial consequence of our convention $\mathcal{M}_{t}^{p}\left(\|f\|_{1},\|g\|_{1}\right):=0$. Normalize $\hat{f}:=f /\|f\|_{1}$ and $\hat{g}:=g /\|g\|_{1}$, and define $\hat{h}$ as in (84). If we can only show $\hat{f}, \hat{g}$ and $\hat{h}$ satisfy hypothesis (7) the desired corollary follows immediately from the conclusion $\int_{M} \hat{h} \geq 1$ of our Main Theorem.

To do this, we employ two standard properties of $p$-means: Hölder's inequality for a two point space, which asserts that for $a, b, c, d>0$ and exponents $p^{-1}+q^{-1}=r^{-1}$ satisfying $p+q \geq 0$, see e.g. Gardner [18, Lemma 10.1],

$$
\begin{gathered}
\mathcal{M}_{t}^{p}(a, b) \mathcal{M}_{t}^{q}(c, d) \geq \mathcal{M}_{t}^{r}(a c, b d), \text { and } \\
\mathcal{M}_{t}^{p}(a, b)^{-1}=\mathcal{M}_{t}^{-p}\left(\frac{1}{a}, \frac{1}{b}\right) .
\end{gathered}
$$

From (83) and hypothesis (8)

$$
\begin{aligned}
\hat{h}(z) & :=\mathcal{M}_{t}^{\frac{p}{1+n p}}\left(\|f\|_{1},\|g\|_{1}\right)^{-1} h(z) \\
& \geq \mathcal{M}_{t}^{\frac{-p}{1+n p}}\left(\frac{1}{\|f\|_{1}}, \frac{1}{\|g\|_{1}}\right) \mathcal{M}_{t}^{p}\left(\frac{f(x)}{v_{1-t}(y, x)}, \frac{g(y)}{v_{t}(x, y)}\right) \\
& \geq \mathcal{M}_{t}^{-1 / n}\left(\frac{\hat{f}(x)}{v_{1-t}(y, x)}, \frac{\hat{g}(y)}{v_{t}(x, y)}\right) \\
& =\mathcal{M}_{t}^{1 / n}\left(\frac{v_{1-t}(y, x)}{\hat{f}(x)}, \frac{v_{t}(x, y)}{\hat{g}(y)}\right)^{-1},
\end{aligned}
$$

where Hölder's inequality has been applied with $q=-p /(1+n p)$ and $r=-1 / n$. Comparing (87) with (7), it is clear that the theorem applies to $\hat{f}, \hat{g}$ and $\hat{h}$ yielding $\|\hat{h}\|_{1} \geq 1$ and hence the desired inequality (9).

The cases $\|f\|_{1}=+\infty$ or $\|g\|_{1}=+\infty$ are handled by approximation from below as at the end of the proof of the Main Theorem. A similarly argument applies to $p=-1 / n$, provided we interpret (9) to mean $\|h\|_{1} \geq$ $\mathcal{M}_{t}^{-\infty}\left(\|f\|_{1},\|g\|_{1}\right):=\min \left\{\|f\|_{1},\|g\|_{1}\right\}$ in this case. This concludes the proof of the corollary. 
Proof of Corollary 1.2. For $(x, y) \in A \times B, z \in Z_{t}(x, y)$ and $d:=d(x, y)$ hypothesis (12) asserts

$$
\begin{aligned}
h(z) & \geq \mathcal{M}_{t}^{0}\left(\left(\frac{S_{k}(d)}{S_{k}((1-t) d)}\right)^{n-1} f(x),\left(\frac{S_{k}(d)}{S_{k}(t d)}\right)^{n-1} g(y)\right) \\
& \geq \mathcal{M}_{t}^{0}\left(\frac{f(x)}{v_{1-t}(y, x)}, \frac{g(y)}{v_{t}(x, y)}\right)
\end{aligned}
$$

by the bound (11) proved in Lemma 2.2. The p-mean corollary (8-9) now asserts $\|h\|_{1} \geq \mathcal{M}_{t}^{0}\left(\|f\|_{1},\|g\|_{1}\right):=\|f\|_{1}^{1-t}\|g\|_{1}^{t}$ establishing (13) and completing the proof.

As a related application of techniques developed herein, we conclude this paper by considering the behaviour of the functional

$$
U(\rho):=\int_{M} A(\rho(x)) d \operatorname{vol}(x)
$$

along the path $\rho_{t} \in L^{1}(M, \mathrm{vol})$ introduced in Sect. 5 to interpolate between $\rho_{0}$ and $\rho_{1}$. In Euclidean space, this path is called the displacement interpolation and the functional (88) is displacement convex if $U\left(\rho_{t}\right)$ is a convex function of $t \in[0,1]$ for every $\rho_{0}, \rho_{1} \in L^{1}(M$, vol ). A sufficient condition for displacement convexity of $U\left(\rho_{t}\right)$ in $\mathbf{R}^{n}$ is that $A:[0, \infty) \longrightarrow \mathbf{R} \cup\{+\infty\}$ satisfy $[25,26]$

$$
\lambda^{n} A\left(\lambda^{-n}\right) \text { is convex non-increasing on } \lambda \in(0, \infty) \text {, with } A(0)=0 .
$$

Typical examples include the entropy $A(\rho)=\rho \log \rho$ and $L^{q}$-norm $A(\rho)=$ $(q-1)^{-1} \rho^{p}$ for $q \geq(n-1) / n$. In very recent work, Otto and Villani [28] gave a formal argument yielding strong versions of such inequalities on a Riemannian manifold. To illustrate the relationship between their Eulerian technique and our Lagrangian approach, the following theorem is offered as a rigorous justification for one of their results: namely, the immediate extension of Euclidean displacement convexity to Ricci non-negative manifolds.

Theorem 6.2 (Displacement convexity on Ricci non-negative manifolds) Let $M$ be a continuously curved Riemannian manifold with Ric $\geq 0$. Fix Borel probability measures $\mu \ll$ vol and $v \ll$ vol on domains $X \subset \subset M$ and $y \subset \subset M$, respectively. Suppose $F_{1}$ pushes $\mu$ forward to $v$, where $F_{t}(x):=\exp _{x}(-t \nabla \phi(x))$ and $\phi \in \mathcal{I}^{c}(\bar{X}, \bar{y})$. Denote the density of $\mu_{t}:=\left(F_{t}\right)_{\#} \mu$ from Proposition 5.4 by $\rho_{t}=d \mu_{t} / d \mathrm{vol}$. Convexity of $U\left(\rho_{t}\right)$ on $t \in[0,1]$ then follows from (88-89).

Proof. For any $t \in[0,1]$, Lemma 5.1 yields $t \phi \in \mathcal{I}^{c}\left(\bar{X}, Z_{t}(\bar{X}, \bar{y})\right)$ and Corollary 4.7 provides a set $K_{t} \subset \mathcal{X}$ of full measure for $\mu_{0}=\mu$ such that 
$\phi$ has a Hessian at each $x \in K_{t}, \rho_{0}(x) \neq 0$ and

$$
U\left(\rho_{t}\right):=\int_{M} A\left(\rho_{t}(z)\right) d \operatorname{vol}(z)=\int_{K_{t}} A\left(\frac{\rho_{0}(x)}{\left(\mathrm{J}_{t}^{1 / n}(x)\right)^{n}}\right)\left(\mathrm{J}_{t}^{1 / n}(x)\right)^{n} d \operatorname{vol}(x),
$$

where $\mathrm{J}_{t}(x) \neq 0$ is defined in (73). Since Ric $\geq 0$ the volume distortion factors satisfy $v_{t}(x, y) \geq 1$ for every $x, y \in M$. Thus for fixed $x \in \cup_{t \in[0,1]} K_{t}$, Lemma 6.1 yields concavity of $\mathrm{J}_{t}^{1 / n}(x)$ on $t \in[0,1]$. Composing this function with the convex non-increasing function (89) yields convexity of the integrand in (90). Although the domain of integration appears to depend on $t$, we derive

$$
U\left(\rho_{(1-s) t+s t^{\prime}}\right) \leq(1-s) U\left(\rho_{t}\right)+s U\left(\rho_{t^{\prime}}\right)
$$

by computing each of the three integrals over the negligibly smaller set $K_{t} \cap K_{t^{\prime}} \cap K_{(1-s) t+s t^{\prime}}$. This proves displacement convexity of $U(\rho)$, and concludes the paper.

\section{References}

1. G. Alberti, L. Ambrosio, A geometrical approach to monotone functions in $\mathbf{R}^{n}$, Math. Z. 230 (1999), 259-316

2. A.D. Aleksandrov, Almost everywhere existence of the second differential of a convex function and some properties of convex surfaces connected with it (in Russian), Uchen. Zap. Leningrad. Gos. Univ., Math. Ser. 6 (1939), 3-35

3. D. Bakry, M. Emery, Diffusions hypercontractives, in: Séminaire de Probabilités XIX, Lecture Notes in Math., 1123, Springer 1985, 177-206

4. V. Bangert, Analytische Eigenschaften konvexer Funktionen auf Riemannschen Mannigfaltigkeiten, J. Reine Angew. Math. 307 (1979), 309-324

5. J-D. Benamou, Y. Brenier, A numerical method for the optimal time-continuous mass transport problem and related problems, pp. 1-11 in: Monge Ampère Equation: Applications to Geometry and Optimization (L.A. Caffarelli, M. Milman, eds.) Contemp. Math. 226, American Mathematical Society, Providence, 1999

6. G. Bianchi, A. Colesanti, C. Pucci, On the second differentiability of convex surfaces, Geom. Dedicata 60 (1996), 39-48

7. R.L. Bishop, R.J. Crittenden, Geometry of Manifolds, Academic Press, New York, 1964

8. S. Bobkov, M. Ledoux, From Brunn-Minkowski to Brascamp-Lieb and to logarithmic Sobolev inequalities, Geom. Funct. Anal. 10 (2000), 1028-1052

9. S. Bobkov, I. Gentil, M. Ledoux, Hypercontractivity of Hamilton-Jacobi equations, to appear in J. Math. Pures Appl.

10. C. Borell, Convex set functions in $d$-space, Period. Math. Hungar. 6 (1975), 111-136

11. H.J. Brascamp, E.H. Lieb, Some inequalities for Gaussian measures and the longrange order of the one-dimensional plasma, pp. 1-14 in: Functional Integration and Its Applications, Proceedings of the International Conference held in London in April 1974 (A.M. Arthur, ed.) Clarendon Press, Oxford, 1975

12. H.J. Brascamp, E.H. Lieb, On extensions of the Brunn-Minkowski and PrékopaLeindler theorems, including inequalities for log concave functions, and with an application to the diffusion equation, J. Funct. Anal. 22 (1976), 366-389 
13. D. Cordero-Erausquin, Inégalité de Prékopa-Leindler sur la sphère, C. R. Acad. Sci. Paris Sér. I. 329 (1999), 789-792

14. D. Cordero-Erausquin, Inégalités géométriques, $\mathrm{PhD}$ thesis, Université de Marne-laVallée, 2000

15. S. Das Gupta, Brunn-Minkowski inequality and its aftermath, J. Multivariate Anal. 10 (1980), 296-318

16. S. Gallot, D. Hulin, J. Lafontaine, Riemannian Geometry, Springer (Universitext), Berlin, 1990

17. W. Gangbo, R.J. McCann, The geometry of optimal transportation, Acta Math. 177 (1996), 113-161

18. R. Gardner, The Brunn-Minkowski inequality, preprint (2000)

19. M. Gromov, V.D. Milman, A topological application of the isoperimetric inequality, Amer. J. Math. 105 (1983), 843-854

20. M. Gromov, Metric Structures for Riemannian and Non-Riemannian Spaces, Birkhäuser, Boston, 1999

21. R. Henstock, A.M. Macbeath, On the measure of sum sets, I. The theorems of Brunn, Minkowski and Lusternik, Proc. London Math. Soc. 3 (1953), 182-194

22. J. Jost, Riemannian Geometry and Geometric Analysis, Springer (Universitext), Berlin, 1998

23. L. Leindler, On a certain converse of Hölder's inequality, Acta Sci. Math. 33 (1972), 217-233

24. B. Maurey, Some deviation inequalities, Geom. Funct. Anal. 1 (1991), 188-197

25. R.J. McCann, A convexity principle for interacting gases and equilibrium crystals, $\mathrm{PhD}$ thesis, Princeton University, 1994

26. R.J. McCann, A convexity principle for interacting gases, Adv. Math. 128 (1997), 153-179

27. R.J. McCann, Polar factorization of maps on Riemannian manifolds, to appear in Geom. Funct. Anal.

28. F. Otto, C. Villani, Generalization of an inequality by Talagrand and links with the logarithmic Sobolev inequality, J. Funct. Anal. 173 (2000), 361-400

29. A. Prékopa, Logarithmic concave measures with application to stochastic programming, Acta Sci. Math. 32 (1971), 301-315

30. A. Prékopa, On logarithmically concave measures and functions, Acta Sci. Math. 34 (1973), 335-343

31. S.T. Rachev, L. Rüschendorf, Mass Transportation Problems, Springer, New York, 1998

32. W. Rudin, Real and Complex Analysis, 3rd ed., McGraw-Hill Book Company, New York, 1987

33. E. Schmidt, Die Brunn-Minkowskische Satz und sein Spiegeltheorem sowie die isoperimetrische Eigenschaft der Kugel in der euklidischen und nichteuklidischen Geometrie, Math. Ann. 120 (1948) 307-422

34. R. Schneider, Convex bodies: the Brunn-Minkowski theory, Cambridge University Press, Cambridge, 1993 